\title{
ON THE APPROXIMATION OF THE FOKKER-PLANCK EQUATION OF THE FINITELY EXTENSIBLE NONLINEAR ELASTIC DUMBBELL MODEL I: A NEW WEIGHTED FORMULATION AND AN OPTIMAL SPECTRAL-GALERKIN ALGORITHM IN TWO DIMENSIONS*
}

\author{
JIE SHEN ${ }^{\dagger}$ AND HAIJUN YU $\ddagger$
}

\begin{abstract}
We propose a new weighted weak formulation for the Fokker-Planck equation of the finitely extensible nonlinear elastic dumbbell model and prove its well-posedness in weighted Sobolev spaces. We also propose simple and efficient semi-implicit time-discretization schemes which are unconditionally stable, i.e., the step size of time marching does not depend on the number of the bases used in configurational space. We then restrict ourselves to the two-dimensional case and construct two Fourier-Jacobi spectral-Galerkin algorithms which enjoy the following properties: (i) they are unconditionally stable, spectrally accurate, and of optimal computational complexity; (ii) they conserve the volume and provide accurate approximation to higher-order moments of the distribution function; and (iii) they can be easily extended to coupled nonhomogeneous systems. Numerical results are presented to show how to choose a proper weight to get the best numerical results of the distribution function and the polymer stress.
\end{abstract}

Key words. finitely extensible nonlinear elastic model, Fokker-Planck equation, spectral method, weighted Sobolev space, Jacobi polynomial

AMS subject classifications. 35K20, 65M70, 65M12, 82xx

DOI. $10.1137 / 100795929$

1. Introduction. The finitely extensible nonlinear elastic (FENE) dumbbell model (cf., for instance, [30, 11, 29] and the references therein) is a well-known coarsegrained model for dilute polymer solutions in which every polymer molecule is modeled by a dumbbell.

Let $\boldsymbol{r}_{1}$ and $\boldsymbol{r}_{2}$ be the position of the two beads of a dumbbell; then the center of the molecule is $\boldsymbol{x}=\left(\boldsymbol{r}_{1}+\boldsymbol{r}_{2}\right) / 2$, and the direction vector of the molecule is $\boldsymbol{q}=\boldsymbol{r}_{2}-\boldsymbol{r}_{1}$. In kinetic theory, a configurational distribution function (CDF) $f(t, \boldsymbol{x}, \boldsymbol{q})$ is used to describe the probability density of finding a molecule at position $\boldsymbol{x}$ and at time $t$ with orientational director $\boldsymbol{q}$. Then, the whole polymer solutions in the background of macroscopic flow field can be described by a macroscopic-mesoscopic coupled system which consists of the Navier-Stokes equations

$$
\begin{aligned}
\frac{\partial \boldsymbol{u}}{\partial t}+\boldsymbol{u} \cdot \nabla_{x} \boldsymbol{u}+\nabla_{x} p & =\frac{1}{\operatorname{Re}} \Delta_{x} \boldsymbol{u}+\frac{\varepsilon}{\operatorname{ReDe}} \nabla_{x} \cdot \boldsymbol{\tau}_{p}, \\
\nabla_{x} \cdot \boldsymbol{u} & =0,
\end{aligned}
$$

${ }^{*}$ Received by the editors May 19, 2010; accepted for publication (in revised form) January 15, 2012; published electronically May 15, 2012. This work is partially supported by AFOSR grant FA9550-08-1-0416 and NFS grant DMS-0915066.

http://www.siam.org/journals/sinum/50-3/79592.html

†School of Mathematical Science, Xiamen University, Xiamen, China, and Department of Mathematics, Purdue University, West Lafayette, IN 47907 (shen7@purdue.edu).

${ }^{\ddagger}$ Corresponding author. LSEC and Institute of Computational Mathematics and Scientific/ Engineering Computing, Academy of Mathematics and Systems Science, Chinese Academy of Sciences, Beijing 100190, China (hyu@lsec.cc.ac.cn). This author's work was also partially supported by the National Science Foundation of China (NSFC 11101413). 
and the Fokker-Planck equation

$$
\frac{\partial f}{\partial t}+\boldsymbol{u} \cdot \nabla_{\boldsymbol{x}} f+\nabla_{\boldsymbol{q}} \cdot(\boldsymbol{K} \cdot q f)=\frac{1}{\mathrm{De}} \nabla_{\boldsymbol{q}} \cdot\left(f \nabla_{\boldsymbol{q}} U\right)+\frac{1}{\mathrm{De}} \Delta_{\boldsymbol{q}} f .
$$

In the above, $\boldsymbol{K}=\left(\nabla_{\boldsymbol{x}} \boldsymbol{u}\right)^{t}$ and $\boldsymbol{\tau}_{p}$ is a polymer stress given by Kramers' expression

$$
\boldsymbol{\tau}_{p}=-\boldsymbol{I}+\int_{\Omega} f \boldsymbol{q} \nabla_{\boldsymbol{q}} U \mathrm{~d} \boldsymbol{q},
$$

where $\Omega$ is the configurational space of $q$ and $U$ is the FENE spring potential

$$
U=-\frac{b}{2} \log \left(1-|\boldsymbol{q}|^{2}\right) .
$$

The system includes four nondimensional constants, $b$, De (Deborah number), Re (Reynolds number), and $\varepsilon$, which are given by

$$
b=\frac{H q_{0}^{2}}{k_{B} \vartheta}, \quad \operatorname{De}=\frac{\zeta q_{0}^{2}}{2 t_{0} k_{B} \vartheta}, \quad \operatorname{Re}=\frac{\rho l_{0} v_{0}}{\eta}, \quad \varepsilon=\frac{n_{0} \zeta q_{0}^{2}}{2 \eta} .
$$

Here $q_{0}$ is the maximum length of dumbbell, $k_{B}$ is the Boltzmann constant, $\vartheta$ is the temperature, $H$ is the FENE spring constant, $\zeta$ is the friction factor of the polymer molecules, $t_{0}$ is the characteristic time of the background flow, $l_{0}$ is the characteristic length of the background flow, $\rho$ is the fluid density, $\eta$ is the fluid viscosity, $n_{0}$ is the number density of polymer molecules, and $v_{0}=l_{0} / t_{0}$ is the characteristic velocity of the background flow.

As a first step toward developing an efficient numerical method for the above coupled system, we shall restrict our attention in this paper to the homogeneous system, i.e., the system in which the $\operatorname{CDF} f(t, \boldsymbol{x}, \boldsymbol{q})$ does not depend on $\boldsymbol{x}$. More precisely, we consider the following Fokker-Planck equation:

$$
\frac{\partial f}{\partial t}+\nabla \cdot(\boldsymbol{K} \cdot \boldsymbol{q} f)=\frac{1}{\mathrm{De}} \nabla \cdot(f \nabla U)+\frac{1}{\mathrm{De}} \Delta f, \quad \boldsymbol{q} \in \Omega=B_{1}(0) \in \mathbb{R}^{d},
$$

where $d=2$ or 3 and $B_{1}(0)$ is a ball centered at the origin with radius 1 . Once we have an efficient solver for the homogeneous Fokker-Planck system (1.7), the coupled Navier-Stokes-Fokker-Planck system (1.1)-(1.5) can be solved by combining a Navier-Stokes solver and the Fokker-Planck solver. (The convection term in (1.3) can be handled with a classical finite difference scheme or a spectral element method; cf., for instance, $[3,6]$ ). Since the spatial variable $\boldsymbol{x}$ does not appear in (1.7), we eliminate the subindex $\boldsymbol{q}$ from the differential operators.

While for given $\boldsymbol{K}$ and $U$ (1.7) is linear, the unbounded FENE potential term $\nabla \cdot(f \nabla U)$ introduces difficulties in both mathematical analysis and numerical approximation. Recently, there have been many mathematical and numerical studies of the homogeneous system (1.7); see, for instance, $[20,7,8,19,29,15,14]$ and the references therein. In particular, some delicate numerical algorithms based on spectral methods were developed recently for the Fokker-Planck equation of the FENE model in $[20,7,15,14,6]$. In fact, the use of spectral methods for the FENE model started as soon as the FENE model was constructed by Warner [30]. Later, Fan [10] improved Warner's method and used the results of the Fokker-Planck equation to verify the approximation of the FENE-P model. Our work is inspired by the recent work of Knezevic and Süli $[15,14]$ and aimed at developing optimal spectral algorithms with 
minimal assumptions on the initial condition. More precisely, our approach will be based on a new weighted weak formulation which allows initial conditions in a much larger functional space, and we construct unconditionally stable semi-implicit schemes in time and spectral-Galerkin algorithms in space with optimal computational complexity while properly handing the pole conditions and unbounded drift.

The main goals of this paper are twofold: (i) introduce a new weighted weak formulation and establish its well-posedness for $b>2$; and (ii) propose simple stable semi-implicit time-discretization schemes and construct two fast Fourier-Jacobi spectral-Galerkin algorithms in the two-dimensional case. The weighted weak formulation proposed here leads to well-posedness in suitable weighted functional spaces and allows us to construct simple and efficient numerical algorithms. The weight in the weak formulation goes directly into the weight of Jacobi polynomials in the numerical implementation and thus leads to linear systems with optimal banded matrices. We hope that this weak formulation and the corresponding estimates we establish here for the homogeneous system will be useful to derive new results for the Navier-StokesFokker-Planck equations where the parameter $b$ is of great interest; see, for example, $[19,17,21,31,4,5]$.

The rest of the paper is organized as follows. In the next section, we introduce the weighted formulation and prove its well-posedness. In section 3, we consider simple semi-implicit time discretization schemes and discuss their stability. Then, we construct two Fourier-Jacobi-Galerkin algorithms with optimal computational complexity in section 4 . We present in section 5 illustrative numerical results to show correctness and effectiveness of our numerical algorithms. We make some concluding remarks in the last section, followed by appendixes containing some tedious calculations on the system matrices of the Jacobi-Galerkin algorithms.

2. A new weak formulation and its well-posedness. We denote $\boldsymbol{D}=$ $\left(\boldsymbol{K}+\boldsymbol{K}^{t}\right) / 2, \boldsymbol{W}=\left(\boldsymbol{K}-\boldsymbol{K}^{t}\right) / 2$, and $M_{s}(\boldsymbol{q})=\left(1-|\boldsymbol{q}|^{2}\right)^{s}$. When $\boldsymbol{W}$ equals zero, (1.7) has a stable equilibrium solution which is the minimizer of the dimensionless free energy (see [30])

$$
A[f]=\int_{\Omega}(f \ln f-f) \mathrm{d} \boldsymbol{q}+\int_{\Omega}\left\{U f-f \frac{\mathrm{De}}{2} \mathbf{D}: \boldsymbol{q} \boldsymbol{q}\right\} \mathrm{d} \boldsymbol{q}
$$

and takes the form

$$
f_{\mathrm{eq}}^{\boldsymbol{D}}(\boldsymbol{q}):=\frac{1}{z} M_{b / 2}(\boldsymbol{q}) \exp ^{\frac{\mathrm{De}}{2} \boldsymbol{D}: \boldsymbol{q} \boldsymbol{q}},
$$

where $z$ is a normalization constant. On the other hand, when $\boldsymbol{D}=0$, the system has a stable steady state solution (see $[12,2])$

$$
f_{b}(\boldsymbol{q}):=\frac{b+2}{2 \pi} M_{b / 2}(\boldsymbol{q}) .
$$

We shall now derive a suitable weak formulation for (1.7). Taking the inner product of (1.7) with a test function $g \in C_{0}^{\infty}(\Omega)$, using (1.5) and integration by parts, we find that the following equation holds:

$$
\left(\frac{\partial f}{\partial t}, g\right)+\frac{b}{\mathrm{De}}\left(\frac{\boldsymbol{q}}{1-|\boldsymbol{q}|^{2}} f, \nabla g\right)+\frac{1}{\mathrm{De}}(\nabla f, \nabla g)=(\boldsymbol{K} \cdot \boldsymbol{q} f, \nabla g) \quad \forall g \in C_{0}^{\infty}(\Omega)
$$

Introducing the change of variable

$$
f(t, \boldsymbol{q})=M_{s}(\boldsymbol{q}) h(t, \boldsymbol{q})
$$


and denoting $(u, v)_{s}=\int_{\Omega} u v M_{s}(\boldsymbol{q}) \mathrm{d} \boldsymbol{q},(2.4)$ becomes

$(2.6)$

$\left(\frac{\partial h}{\partial t}, g\right)_{s}+\frac{b-2 s}{\mathrm{De}}\left(\frac{\boldsymbol{q}}{1-|\boldsymbol{q}|^{2}} h, \nabla g\right)_{s}+\frac{1}{\mathrm{De}}(\nabla h, \nabla g)_{s}=(\boldsymbol{K} \cdot \boldsymbol{q} h, \nabla g)_{s} \quad \forall g \in C_{0}^{\infty}(\Omega)$.

Now let us introduce some functional spaces which will be used in what follows:

$$
\begin{aligned}
& L_{s}^{2}(\Omega) \equiv L^{2}\left(\Omega, M_{s}\right):=\left\{g:\|g\|_{L_{s}^{2}}^{2} \equiv\|g\|_{L^{2}\left(\Omega, M_{s}\right)}^{2}:=\int_{\Omega}|g|^{2} M_{s}(\boldsymbol{q}) \mathrm{d} \boldsymbol{q}<\infty\right\}, \\
& X_{s}(\Omega) \equiv H^{1,2}\left(\Omega, M_{s}\right):=\left\{g:\|g\|_{X_{s}}^{2} \equiv\|g\|_{H^{1,2}\left(\Omega, M_{s}\right)}^{2}:=\|g\|_{L^{2}\left(\Omega, M_{s}-2\right)}^{2}\right.\left.+\sum_{\alpha=1}^{d}\left\|\partial_{\alpha} g\right\|_{L^{2}\left(\Omega, M_{s}\right)}^{2}<\infty\right\} \\
& W^{1,2}\left(\Omega, M_{s}\right):=\left\{g:\|g\|_{W^{1,2}\left(\Omega, M_{s}\right)}^{2}:=\|g\|_{L^{2}\left(\Omega, M_{s}\right)}^{2}\right.\left.+\sum_{\alpha=1}^{d}\left\|\partial_{\alpha} g\right\|_{L^{2}\left(\Omega, M_{s}\right)}^{2}<\infty\right\} \\
& L^{2}(0, T ; F):=\left\{g(t, \boldsymbol{q}):\|g\|_{L^{2}(0, T ; F)}^{2}:=\int_{0}^{T}\|g(t, \cdot)\|_{F}^{2} \mathrm{~d} t<\infty\right\}
\end{aligned}
$$

and

$$
L^{\infty}(0, T ; F):=\left\{g(t, \boldsymbol{q}):\|g\|_{L^{\infty}(0, T ; F)}:=\operatorname{ess}_{\sup _{0 \leq t \leq T}}\|g(t, \cdot)\|_{F}<\infty\right\} .
$$

Remark 2.1. The weighted Sobolev type spaces $W^{1,2}\left(\Omega, M_{s}\right)$ and $H^{1,2}\left(\Omega, M_{s}\right)$ are well studied in [16]. For $s>1$, it is proved that $W_{0}^{1,2}\left(\Omega ; M_{s}\right)=H^{1,2}\left(\Omega ; M_{s}\right)=$ $W^{1,2}\left(\Omega ; M_{s}\right)$, i.e., $C_{0}^{\infty}(\Omega)$ is dense in $H^{1,2}\left(\Omega, M_{s}\right)$, and the $W^{1,2}\left(\Omega, M_{s}\right)$ norm and $H^{1,2}\left(\Omega, M_{s}\right)$ are equivalent. (See [16, Proposition 9.6 and Theorem 9.7, p. 68].)

The new weak formulation for $(2.6)$ is

given $h_{0}(\boldsymbol{q}) \in L^{2}\left(\Omega, M_{s}\right)$, find $h \in L^{2}\left(0, T ; X_{s}(\Omega)\right) \cap L^{\infty}\left(0, T ; L^{2}\left(\Omega, M_{s}\right)\right)$ such that

$$
\left(\frac{\partial h}{\partial t}, g\right)_{s}+\frac{b-2 s}{\operatorname{De}}\left(\frac{\boldsymbol{q}}{1-|\boldsymbol{q}|^{2}} h, \nabla g\right)_{s}+\frac{1}{\operatorname{De}}(\nabla h, \nabla g)_{s}=(\boldsymbol{K} \cdot \boldsymbol{q} h, \nabla g)_{s} \quad \forall g \in X_{s}(\Omega),
$$
$h(0, \boldsymbol{q})=h_{0}(\boldsymbol{q})$.

Based on (2.5), the corresponding weak formulation for the $\mathrm{CDF} f$ is

$$
\text { given } f_{0}(\boldsymbol{q}) \in L^{2}\left(\Omega, M_{-s}\right) \text {, find } f \in L^{2}\left(0, T ; X_{s}^{*}(\Omega)\right) \cap L^{\infty}\left(0, T ; L^{2}\left(\Omega, M_{-s}\right)\right)
$$

such that

$$
\begin{aligned}
& \left(\frac{\partial f}{\partial t}, g\right)_{-s}+\frac{b-2 s}{\mathrm{De}}\left(\frac{\boldsymbol{q}}{1-|\boldsymbol{q}|^{2}} f, \nabla \frac{g}{M_{s}}\right)+\frac{1}{\mathrm{De}}\left(\nabla \frac{f}{M_{s}}, \nabla \frac{g}{M_{s}}\right)_{s} \\
& =\left(\boldsymbol{K} \cdot \boldsymbol{q} f, \nabla \frac{g}{M_{s}}\right) \quad \forall g \in X_{s}^{*}(\Omega), \\
& f(0, \boldsymbol{q})=f_{0}(\boldsymbol{q}),
\end{aligned}
$$


where

$$
X_{s}^{*}(\Omega):=\left\{f:\|f\|_{X_{s}^{*}}^{2}=\int_{\Omega}|f|^{2} M_{-s}(\boldsymbol{q}) \mathrm{d} \boldsymbol{q}+\int_{\Omega}\left|\nabla \frac{f}{M_{s}} \cdot \nabla \frac{f}{M_{s}}\right| M_{s} \mathrm{~d} \boldsymbol{q}<\infty\right\} .
$$

The main result in this section is the following.

THEOREM 2.1. Given $1<s \leqslant b / 2$ and $h_{0}(\boldsymbol{q}) \in L^{2}\left(\Omega, M_{s}\right)$, there exists a unique solution $h$ for the problem (2.12). Moreover, there exists a positive constant $C$, depending only on $T, b, s$, De, and $\boldsymbol{K}$, such that

$$
\max _{0 \leqslant t \leqslant T}\|h(t, \cdot)\|_{L^{2}\left(\Omega, M_{s}\right)}^{2}+\|h\|_{L^{2}\left(0, T ; X_{s}\right)}^{2} \leqslant C\left\|h_{0}\right\|_{L^{2}\left(\Omega, M_{s}\right)}^{2} .
$$

A straightforward consequence of the above theorem is the following corollary.

Corollary 2.1. Given $1<s \leqslant b / 2$, and $f_{0}(\boldsymbol{q}) \in L^{2}\left(\Omega, M_{-s}\right)$, there exists a unique solution $f$ for the problem (2.13). Moreover, there exists a positive constant $C$, depending only on $T, b, s$, De, and $\boldsymbol{K}$, such that

$$
\max _{0 \leqslant t \leqslant T}\|f(t, \cdot)\|_{L^{2}\left(\Omega, M_{-s}\right)}^{2}+\|f\|_{L^{2}\left(0, T ; X_{s}^{*}\right)}^{2} \leqslant C\left\|f_{0}\right\|_{L^{2}\left(\Omega, M_{-s}\right)}^{2} .
$$

Remark 2.2. For $1<s$, no boundary condition is required, since $C_{0}^{\infty}(\Omega)$ is dense in $W^{1,2}\left(\Omega, M_{s}\right)$. The results of Theorem 2.1 and Corollary 2.1 are consistent with the results in [19,13]. In [15], the case $s=b / 2$ was considered. In this particular case, the weak formulation in [15] is equivalent to (2.13).

We only need to prove Theorem 2.1. To this end, we need to establish some basic estimates and lemmas.

2.1. Basic estimates. We define two bilinear forms in $X_{s}(\Omega) \times X_{s}(\Omega)$ by

$$
\begin{aligned}
& A[h, g]=\frac{b-2 s}{\mathrm{De}}\left(\frac{\boldsymbol{q}}{1-|\boldsymbol{q}|^{2}} h, \nabla g\right)_{s}+\frac{1}{\mathrm{De}}(\nabla h, \nabla g)_{s}, \\
& B[h, g]=A[h, g]-(\boldsymbol{K} \cdot \boldsymbol{q} h, \nabla g)_{s} .
\end{aligned}
$$

Then, we can rewrite $(2.12)$ as

$$
\left(\frac{\partial h}{\partial t}, g\right)_{s}+B[h(t, \cdot), g]=0 \quad \forall g \in X_{s}(\Omega) .
$$

We start by establishing the continuity and coercivity of the two bilinear forms $A$ and $B$.

Lemma 2.1. Given $1<s \leq b / 2$. Let $C_{a}=\frac{1}{\mathrm{De}}+\frac{b-2 s}{\mathrm{De}}, C_{b}=\frac{1}{\mathrm{De}}+\frac{b-2 s}{\mathrm{De}}+|\boldsymbol{K}|_{\infty}$ with $|\boldsymbol{K}|_{\infty}=\max _{i, j}\left|K_{i j}\right|$. Then

$$
A[h, g] \leqslant C_{a}\|h\|_{X_{s}}\|\nabla g\|_{s} \leqslant C_{a}\|h\|_{X_{s}}\|g\|_{X_{s}} \quad \forall h, g \in X_{s}(\Omega)
$$

and

$$
B[h, g] \leqslant C_{b}\|h\|_{X_{s}}\|\nabla g\|_{s} \leqslant C_{b}\|h\|_{X_{s}}\|g\|_{X_{s}} \quad \forall h, g \in X_{s}(\Omega),
$$

and there exist positive constants $\gamma_{a}, \beta_{a}, \gamma_{b}, \beta_{b}$ such that

$$
\begin{array}{ll}
A[h, h]+\gamma_{a}\|h\|_{s}^{2} \geqslant \beta_{a}\|h\|_{X_{s}}^{2} & \forall h \in X_{s}(\Omega), \\
B[h, h]+\gamma_{b}\|h\|_{s}^{2} \geqslant \beta_{b}\|h\|_{X_{s}}^{2} & \forall h \in X_{s}(\Omega) .
\end{array}
$$


Proof. The proof for the case $s=b / 2$ is trivial, so we consider only $1<s<b / 2$ below.

First, by using the Cauchy-Schwarz inequality, we have

$$
\begin{aligned}
A[h, g] & \leqslant \frac{b-2 s}{\mathrm{De}}\left\|h \frac{\boldsymbol{q}}{1-|\boldsymbol{q}|^{2}}\right\|_{s}\|\nabla g\|_{s}+\frac{1}{\mathrm{De}}\|\nabla h\|_{s}\|\nabla g\|_{s} \\
& \leqslant C_{a}\|h\|_{X_{s}}\|\nabla g\|_{s} \leqslant C_{a}\|h\|_{X_{s}}\|g\|_{X_{s}}
\end{aligned}
$$

and

$$
B[h, g] \leqslant C_{a}\|h\|_{X_{s}}\|\nabla g\|_{s}+|\boldsymbol{K}|_{\infty}\|h\|_{s}\|\nabla g\|_{s} \leqslant C_{b}\|h\|_{X_{s}}\|\nabla g\|_{s} \leqslant C_{b}\|h\|_{X_{s}}\|g\|_{X_{s}} .
$$

Next, we observe that

$$
\begin{aligned}
\left(\frac{\boldsymbol{q}}{1-|\boldsymbol{q}|^{2}} h, \nabla h\right)_{s}= & \frac{1}{2} \int_{\partial \Omega} \frac{h^{2}}{1-|\boldsymbol{q}|^{2}} M_{s} \mathrm{~d} S-\frac{d}{2} \int_{\Omega} \frac{h^{2}}{1-|\boldsymbol{q}|^{2}} M_{s} \mathrm{~d} \boldsymbol{q} \\
& +(s-1) \int_{\Omega} \frac{|\boldsymbol{q}|^{2} h^{2}}{\left(1-|\boldsymbol{q}|^{2}\right)^{2}} M_{s} \mathrm{~d} \boldsymbol{q} \\
= & (s-1)\|\boldsymbol{q} h\|_{s-2}^{2}-\frac{d}{2}\|h\|_{s-1}^{2} .
\end{aligned}
$$

Setting

$$
\gamma(s)=\frac{d}{4}\left(1+\frac{(s-1)}{d}+\frac{d}{4(s-1)}\right), \quad \lambda(\gamma)=(s-1)\left(1-\frac{\gamma(s)}{\gamma}\right),
$$

it is easy to verify that for $\gamma>\gamma(s)$, we have

$$
\gamma\left(1-|\boldsymbol{q}|^{2}\right)^{2}-\frac{d}{2}\left(1-|\boldsymbol{q}|^{2}\right)+(s-1)|\boldsymbol{q}|^{2} \geqslant \lambda(\gamma)>0, \quad|\boldsymbol{q}|^{2} \in[0,1] .
$$

Therefore, we derive from the above that for $\gamma>\gamma(s)$,

$$
\left(\frac{\boldsymbol{q}}{1-|\boldsymbol{q}|^{2}} h, \nabla h\right)_{s}+\gamma\|h\|_{s}^{2}=(s-1)\|\boldsymbol{q} h\|_{s-2}^{2}-\frac{d}{2}\|h\|_{s-1}^{2}+\gamma\|h\|_{s}^{2} \geqslant \lambda(\gamma)\|h\|_{s-2}^{2}
$$

which we rewrite as, for $\gamma>\gamma(s)$,

$$
\frac{b-2 s}{\operatorname{De}}\left(\frac{\boldsymbol{q}}{1-|\boldsymbol{q}|^{2}} h, \nabla h\right)_{s}+\frac{(b-2 s) \gamma}{\operatorname{De}}\|h\|_{s}^{2} \geqslant \frac{(b-2 s) \lambda(\gamma)}{\operatorname{De}}\|h\|_{s-2}^{2} .
$$

Hence, for $\gamma>\gamma(s)$, and $\beta_{a}=\min \left\{\frac{1}{\mathrm{De}}, \frac{b-2 s}{\mathrm{De}} \lambda(\gamma)\right\}$, we have

$$
A[h, h]+\frac{(b-2 s) \gamma}{\mathrm{De}}\|h\|_{s}^{2} \geqslant \frac{(b-2 s) \lambda(\gamma)}{\mathrm{De}}\|h\|_{s-2}^{2}+\frac{1}{\mathrm{De}}(\nabla h, \nabla h)_{s} \geqslant \beta_{a}\|h\|_{X_{s}}^{2},
$$

which implies that

$$
A[h, h]+\gamma_{a}\|h\|_{s}^{2} \geqslant \beta_{a}\|h\|_{X_{s}}^{2},
$$

where

$$
\gamma_{a}>\gamma_{\min }=\frac{b-2 s}{\operatorname{De}} \gamma(s), \quad \beta_{a}=\min \left\{\frac{1}{\mathrm{De}}, \frac{b-2 s}{\operatorname{De}} \lambda\left(\frac{\gamma_{a} \mathrm{De}}{b-2 s}\right)\right\} .
$$


For the bilinear form $B[h, g]$, we first use the Cauchy-Schwarz inequality to obtain

$$
(\boldsymbol{K} \cdot \boldsymbol{q} h, \nabla h)_{s} \leqslant \frac{\mathrm{De}}{2}|\boldsymbol{K}|_{\infty}^{2}\|h\|_{s}^{2}+\frac{1}{2 \mathrm{De}}\|\nabla h\|_{s}^{2} .
$$

Setting $c_{0}=\operatorname{De}|\boldsymbol{K}|_{\infty}^{2}$, we find

$$
B[h, h] \geqslant \frac{b-2 s}{\operatorname{De}}\left(\frac{\boldsymbol{q}}{1-|\boldsymbol{q}|^{2}} h, \nabla h\right)_{s}-\frac{c_{0}}{2}\|h\|_{s}^{2}+\frac{1}{2 \mathrm{De}}\|\nabla h\|_{s}^{2} .
$$

Then, by using inequality (2.25), we get

$$
B[h, h]+\left(\frac{(b-2 s) \gamma}{\mathrm{De}}+\frac{c_{0}}{2}\right)\|h\|_{s}^{2} \geqslant \frac{(b-2 s) \lambda(\gamma)}{\mathrm{De}}\|h\|_{s-2}^{2}+\frac{1}{2 \mathrm{De}}\|\nabla h\|_{s}^{2} .
$$

Therefore, we have

$$
B[h, h]+\gamma_{b}\|h\|_{s}^{2} \geqslant \beta_{b}\|h\|_{X_{s}}^{2}
$$

where

$$
\gamma_{b}>\frac{(b-2 s) \gamma(s)}{\mathrm{De}}+\frac{c_{0}}{2} \quad \text { and } \quad \beta_{b}=\min \left\{\frac{1}{2 \mathrm{De}}, \frac{b-2 s}{\operatorname{De}} \lambda\left(\frac{\left(\gamma_{b}-c_{0} / 2\right) \mathrm{De}}{b-2 s}\right)\right\} .
$$

Next, we use a standard Galerkin approximation procedure (see, e.g., [9]). Since the embedding of $X_{s}$ in $L^{2}\left(\Omega, M_{s}\right)$ is compact, there exists a complete set of appropriately normalized eigenfunctions $\left\{\phi_{k}\right\}_{k=1}^{\infty}$ of the operator $-M_{-s} \nabla \cdot\left(M_{s} \nabla\right)$ in $X_{s}$, which is also a set of orthogonal bases in both $X_{s}$ and $L^{2}\left(\Omega ; M_{s}\right)$. The Galerkin approximation of $(2.12)$ is as follows: Find $h_{m}(t, \cdot) \in H_{m}=\operatorname{span}\left\{\phi_{k}, k=1, \ldots, m\right\}$ such that

$$
\left(h_{m}^{\prime}, g\right)_{s}+B\left[h_{m}, g\right]=0 \quad \forall g \in H_{m},
$$

where $h_{m}^{\prime}$ is a shorthand notation of $\frac{\partial h_{m}}{\partial t}$. And

$$
\left(h_{m}(0, \cdot), \phi_{k}\right)_{s}=\left(h_{0}, \phi_{k}\right)_{s}, \quad k=1, \ldots, m .
$$

LEMMA 2.2. The problem (2.28)-(2.29) admits a unique solution, and there exists a constant $C$, depending only on $T, b, s, \mathrm{De}$, and $\mathbf{K}$, such that

$$
\max _{0 \leqslant t \leqslant T}\left\|h_{m}\right\|_{s}^{2}+\left\|h_{m}\right\|_{L^{2}\left(0, T ; X_{s}\right)}^{2}+\left\|h_{m}^{\prime}\right\|_{L^{2}\left(0, T ; X_{s}^{\prime}\right)}^{2} \leqslant C\left\|h_{0}\right\|_{s}^{2}, \quad m=1,2, \ldots,
$$

where $X_{s}^{\prime}$ is the dual space of $X_{s}(\Omega)$.

Proof. Writing $h_{m}(t, \boldsymbol{q})=\sum_{k=1}^{m} b_{m, k}(t) \phi_{k}(\boldsymbol{q})$, and taking $g=\phi_{k}(k=1, \ldots, m)$ in (2.28), we arrive at a system of linear ODEs for the coefficients $\left\{b_{m, k}(t)\right\}_{k=1}^{m}$. It is then clear from the theory of linear ODEs that this system admits a unique solution in the interval $[0, T]$.

Next, we take $g=h_{m}(t, \cdot)$ in $(2.28)$ to obtain

$$
\left(h_{m}^{\prime}, h_{m}\right)_{s}+B\left[h_{m}, h_{m}\right]=0 \quad \text { a.e. } \quad 0<t \leqslant T .
$$

Thanks to the property (2.21), we get

$$
\frac{\mathrm{d}}{\mathrm{d} t}\left\|h_{m}\right\|_{s}^{2}+2 \beta_{b}\left\|h_{m}\right\|_{X_{s}}^{2} \leqslant 2 \gamma_{b}\left\|h_{m}\right\|_{s}^{2} .
$$


Applying Gronwall's inequality, we find

$$
\max _{0 \leqslant t \leqslant T}\left\|h_{m}(t)\right\|_{s}^{2} \leqslant e^{2 \gamma_{b} T}\left\|h_{0}\right\|_{s}^{2} .
$$

Now integrating (2.31) from 0 to $T$, and using the inequality above, we obtain

$$
\left\|h_{m}\right\|_{L^{2}\left(0, T ; X_{s}\right)}^{2} \leqslant\left(1+T\left(e^{2 \gamma_{b} T}-1\right)\right) / 2 \beta_{b}\left\|h_{0}\right\|_{s}^{2} .
$$

It remains to show that $\left\|h_{m}^{\prime}\right\|_{L^{2}\left(0, T ; X_{s}^{\prime}\right)}^{2}$ is bounded. For any $g \in X_{s}$ with $\|g\|_{X_{s}}=$ 1 , we write $g=g_{1}+g_{2}$, where $g_{1} \in H_{m}$ and $\left(g_{2}, \phi_{k}\right)_{X_{s}}=0, k=1, \ldots, m$. Obviously,

$$
\left\|\nabla g_{1}\right\|_{s} \leqslant\|\nabla g\|_{s} \leqslant\|g\|_{X_{s}}=1
$$

and

$$
\left(h_{m}^{\prime}, g\right)_{s}=\left(h_{m}^{\prime}, g_{1}\right)_{s}=-B\left[h_{m}, g_{1} ; t\right] \leqslant C_{b}\left\|h_{m}\right\|_{X_{s}}\left\|\nabla g_{1}\right\|_{s} \leqslant C_{b}\left\|h_{m}\right\|_{X_{s}},
$$

which implies

$$
\left\|h_{m}^{\prime}\right\|_{X_{s}^{\prime}} \leqslant C_{b}\left\|h_{m}\right\|_{X_{s}} .
$$

We then drive from the above and (2.32) that

$$
\int_{0}^{T}\left\|h_{m}^{\prime}\right\|_{X_{s}^{\prime}}^{2} \mathrm{~d} t \leqslant C_{b} \int_{0}^{T}\left\|h_{m}\right\|_{X_{s}}^{2} \mathrm{~d} t \leq C\left\|h_{0}\right\|_{s}^{2} .
$$

This completes the proof.

We shall need the following lemma, which is a particular case of a general theorem of interpolation by Lions and Magenes [18] (see also [28] and [9]).

Lemma 2.3. For $1<s$, if $h \in L^{2}\left(0, T ; X_{s}(\Omega)\right)$ and $h^{\prime} \in L^{2}\left(0, T ; X_{s}^{\prime}(\Omega)\right)$, then we have

$$
h \in C\left([0, T] ; L^{2}\left(\Omega, M_{s}\right)\right) .
$$

The mapping $t \rightarrow\|h\|_{s}^{2}$ is absolutely continuous with

$$
\frac{\mathrm{d}}{\mathrm{d} t}\|h\|_{s}^{2}=2\left(h^{\prime}(t, \cdot), h(t, \cdot)\right)_{s} \text { for a.e. } 0 \leqslant t \leqslant T .
$$

2.2. Proof of Theorem 2.1. With the above lemmas, the proof of Theorem 2.1 can be carried out following a standard procedure for parabolic equations as described in, e.g., [9]. For the reader's convenience, we sketch the main steps below.

The uniqueness is a direct consequence of Lemma 2.1, so we only need to prove the existence.

Step 1. According to the energy estimate (2.30), the sequence $\left\{h_{m}\right\}_{m=1}^{\infty}$ is bounded in $L^{2}\left(0, T ; X_{s}\right)$ and $\left\{h_{m}^{\prime}\right\}_{m=1}^{\infty}$ is bounded in $L^{2}\left(0, T ; X_{s}^{\prime}\right)$. Consequently, there exists a subsequence $\left\{h_{m_{l}}\right\}_{l=1}^{\infty},\left\{h_{m_{l}}^{\prime}\right\}_{l=1}^{\infty}$ and a function $h \in L^{2}\left(0, T ; X_{s}\right)$ with $h^{\prime} \in L^{2}\left(0, T ; X_{s}^{\prime}\right)$ such that

$$
h_{m_{l}} \rightarrow h \quad \text { weakly in } L^{2}\left(0, T ; X_{s}\right), \quad h_{m_{l}}^{\prime} \rightarrow h^{\prime} \quad \text { weakly in } L^{2}\left(0, T ; X_{s}^{\prime}\right) .
$$

Step 2. Fix an integer $N$ and choose a function $g \in C^{1}\left([0, T] ; H_{N}\right)$ having the form

$$
g(t, \boldsymbol{q})=\sum_{k=1}^{N} b_{k}(t) \phi_{k}(\boldsymbol{q})
$$


where $\left\{b_{k}(t)\right\}_{k=1}^{N}$ are (arbitrarily) given smooth functions. Then in (2.28), we let $m \geqslant N$ and integrate with respect to $t$

$$
\int_{0}^{T}\left(h_{m}^{\prime}, g\right)_{s}+B\left[h_{m}, g\right] \mathrm{d} t=0
$$

Letting $m=m_{l} \rightarrow \infty$, we find

$$
\int_{0}^{T}\left(h^{\prime}, g\right)_{s}+B[h, g] \mathrm{d} t=0 .
$$

The above equation holds for any function $g$ in $L^{2}\left(0, T ; H_{N}\right)$, since functions of the form $(2.33)$ are dense in $L^{2}\left(0, T ; H_{N}\right)$. Hence, for any $g \in L^{2}\left(0, T ; X_{s}\right)$, we have

$$
\left(h^{\prime}, g\right)_{s}+B[h, g ; t]=0 \quad \text { a.e. } 0 \leqslant t \leqslant T .
$$

We then derive from Lemma 2.3 that $h \in C\left([0, T] ; L^{2}\left(\Omega, M_{s}\right)\right)$.

Step 3. In order to prove $h(0, \boldsymbol{q})=h_{0}(\boldsymbol{q})$, we first note from (2.35) that

$$
-\int_{0}^{T}\left(h, g^{\prime}\right)_{s}+B[h, g] \mathrm{d} t=(h(0, \boldsymbol{q}), g(0, \boldsymbol{q}))_{s}
$$

for each $g \in C^{1}\left([0, T] ; X_{s}\right)$ with $g(T, \boldsymbol{q})=0$. Similarly, from (2.34) we deduce

$$
-\int_{0}^{T}\left(h_{m}, g^{\prime}\right)_{s}+B\left[h_{m}, g\right] \mathrm{d} t=\left(h_{m}(0, \boldsymbol{q}), g(0, \boldsymbol{q})\right)_{s} .
$$

Letting $m=m_{l} \rightarrow \infty$, we find

$$
-\int_{0}^{T}\left(h, g^{\prime}\right)_{s}+B[h, g] \mathrm{d} t=\left(h_{0}(\boldsymbol{q}), g(0, \boldsymbol{q})\right)_{s} .
$$

As $g(0, \boldsymbol{q})$ is arbitrary, comparing $(2.37)$ and $(2.38)$, we conclude that $h(0, \boldsymbol{q})=h_{0}(\boldsymbol{q})$.

3. Semidiscretization schemes in time and their stability. We construct in this section efficient semidiscretization schemes in time and discuss their stability. It is clear that in order to have good stability, the terms in the bilinear form $A[\cdot, \cdot]$ should be treated implicitly. On the other hand, we observe from the proof of wellposedness in the last section that the term $\nabla \cdot(\boldsymbol{K} \cdot \boldsymbol{q} h)$ is not dissipative so it does not help on the stability to treat it implicitly. In fact, the proof of well-posedness in the last section suggests that it can be treated explicitly without affecting the stability. Such a semi-implicit treatment not only allows us to prove unconditional stability but also enables us to construct simple and fast spectral algorithms in the next section.

3.1. A first-order semi-implicit scheme. We start with a first-order semiimplicit scheme for (2.12).

Given $h^{0} \in L^{2}\left(\Omega, M_{s}\right)$, for $n=0,1, \ldots$, find $h^{n+1}(\boldsymbol{q}) \in X_{s}(\Omega)$ such that

$$
\begin{gathered}
\left(\frac{h^{n+1}-h^{n}}{\Delta t}, g\right)_{s}+\frac{b-2 s}{\mathrm{De}}\left(\frac{\boldsymbol{q}}{1-|\boldsymbol{q}|^{2}} h^{n+1}, \nabla g\right)_{s}+\frac{1}{\mathrm{De}}\left(\nabla h^{n+1}, \nabla g\right)_{s} \\
=\left(\boldsymbol{K} \cdot \boldsymbol{q} h^{n}, \nabla g\right)_{s} \quad \forall g \in X_{s}(\Omega) .
\end{gathered}
$$

Theorem 3.1. Let $1<s \leq b / 2, h^{0} \in L^{2}\left(\Omega, M_{s}\right)$, and $\gamma_{\min }$ be as defined in (2.26). Then, for $\Delta t>0$ when $s=b / 2$, and for $0<\Delta t<1 / \gamma_{\text {min }}$ when $1<s<b / 2$, the 
scheme (3.1) admits a unique solution $h^{n+1}(\boldsymbol{q}) \in X_{s}(\Omega)$ for $n=0,1, \ldots$ Moreover, we have

$$
\left\|h^{m+1}\right\|_{b / 2}^{2}+\Delta t \sum_{n=0}^{m}\left\|\frac{h^{n+1}-h^{n}}{\sqrt{\Delta t}}\right\|_{b / 2}^{2}+\frac{\Delta t}{\operatorname{De}} \sum_{n=0}^{m}\left\|\nabla h^{n+1}\right\|_{b / 2}^{2} \leqslant e^{c_{0} m \Delta t}\left\|h^{0}\right\|_{b / 2}^{2}
$$

for $s=b / 2$ with any $\Delta t$ and

$$
\begin{aligned}
\left\|h^{m+1}\right\|_{s}^{2}+\Delta t \sum_{n=0}^{m}\left\|\frac{h^{n+1}-h^{n}}{\sqrt{\Delta t}}\right\|_{s}^{2} & +\frac{\Delta t}{\operatorname{De}} \sum_{n=0}^{m}\left\|\nabla h^{n+1}\right\|_{s}^{2} \\
& +2 \beta(\gamma) \Delta t \sum_{n=0}^{m}\left\|h^{n+1}\right\|_{s-2}^{2} \leqslant e^{c_{1} m \Delta t}\left\|h^{0}\right\|_{s}^{2}
\end{aligned}
$$

for $1<s<b / 2$ with $\Delta t<1 / 6 \gamma$ and $\gamma>\gamma_{\min }$. Here $c_{0}=\operatorname{De}|\mathbf{K}|_{\infty}^{2}, c_{1}=3 c_{0} / 2+3 \gamma$, $\beta(\gamma)=\frac{b-2 s}{\mathrm{De}} \lambda\left(\frac{\mathrm{De} \gamma}{b-2 s}\right)$.

Proof. We rewrite (3.1) as

$$
\bar{A}\left[h^{n+1}, g\right]=\left\langle h^{n}, g\right\rangle \quad \forall g \in X_{s}(\Omega),
$$

where

$$
\bar{A}[u, v]=\frac{1}{\Delta t}(u, v)_{s}+A[u, v]
$$

and

$$
\langle u, v\rangle=(\boldsymbol{K} \cdot \boldsymbol{q} u, \nabla v)_{s}+\frac{1}{\Delta t}(u, v)_{s} .
$$

It is obvious that the problem (3.4) admits a unique solution for any $\Delta t>0$ when $s=b / 2$.

We now consider $1<s<b / 2$. It is easy to verify that $\left|\left\langle h^{n}, g\right\rangle\right| \leq C\|g\|_{X_{s}}$. On the other hand, we derive from the estimates (2.18) and (2.20) that

$$
\bar{A}[u, v] \leqslant\left(\frac{1}{\Delta t}+C_{a}\right)\|u\|_{X_{s}}\|v\|_{X_{s}}
$$

and

$$
\bar{A}[u, u]+\left(\gamma_{a}-\frac{1}{\Delta t}\right)\|u\|_{s} \geqslant \beta_{a}\|u\|_{X_{s}}, \quad \gamma_{a}>\gamma_{\min } .
$$

Thanks to the generalized Lax-Milgram theorem, the problem (3.4) admits a unique solution when $\Delta t<1 / \gamma_{\min }$.

Next, we prove the estimate when $s=b / 2$.

Taking $g=h^{n+1}$ in (3.1) and using the identity

$$
2(\alpha-\beta, \alpha)=(\alpha, \alpha)-(\beta, \beta)+(\alpha-\beta, \alpha-\beta),
$$

we obtain

$$
\begin{aligned}
\frac{1}{2 \Delta t}\left(\left\|h^{n+1}\right\|_{b / 2}^{2}-\left\|h^{n}\right\|_{b / 2}^{2}+\| h^{n+1}-\right. & \left.h^{n} \|_{b / 2}^{2}\right) \\
& +\frac{1}{\operatorname{De}}\left\|\nabla h^{n+1}\right\|_{b / 2}^{2}=\left(\boldsymbol{K} \cdot \boldsymbol{q} h^{n}, \nabla h^{n+1}\right)_{b / 2} .
\end{aligned}
$$


Then, by using the Cauchy-Schwarz inequality on the term on the right-hand side, we find

$\frac{1}{2 \Delta t}\left(\left\|h^{n+1}\right\|_{b / 2}^{2}-\left\|h^{n}\right\|_{b / 2}^{2}+\left\|h^{n+1}-h^{n}\right\|_{b / 2}^{2}\right)+\frac{1}{2 \mathrm{De}}\left\|\nabla h^{n+1}\right\|_{b / 2}^{2} \leqslant \frac{\mathrm{De}}{2}|\boldsymbol{K}|_{\infty}^{2}\left\|h^{n}\right\|_{b / 2}^{2}$,

which we can rewrite with $c_{0}=\operatorname{De}|\boldsymbol{K}|_{\infty}^{2}$ as

$$
\left\|h^{n+1}\right\|_{b / 2}^{2}+\left\|h^{n+1}-h^{n}\right\|_{b / 2}^{2}+\frac{\Delta t}{\mathrm{De}}\left\|\nabla h^{n+1}\right\|_{b / 2}^{2} \leqslant\left(1+c_{0} \Delta t\right)\left\|h^{n}\right\|_{b / 2}^{2} .
$$

Then, summing up the above relation for $n=0,1, \ldots, m$, we get

$\left\|h^{m+1}\right\|_{b / 2}^{2}+\sum_{n=0}^{m}\left\|h^{n+1}-h^{n}\right\|_{b / 2}^{2}+\frac{\Delta t}{\operatorname{De}} \sum_{n=0}^{m}\left\|\nabla h^{n+1}\right\|_{b / 2}^{2} \leqslant\left\|h^{0}\right\|_{b / 2}^{2}+c_{0} \Delta t \sum_{n=0}^{m}\left\|h^{n}\right\|_{b / 2}^{2}$.

By applying a discrete Gronwall lemma, we deduce that

$$
\left\|h^{m+1}\right\|_{b / 2}^{2}+\sum_{n=0}^{m}\left\|h^{n+1}-h^{n}\right\|_{b / 2}^{2}+\frac{\Delta t}{\operatorname{De}} \sum_{n=0}^{m}\left\|\nabla h^{n+1}\right\|_{b / 2}^{2} \leqslant e^{c_{0} m \Delta t}\left\|h^{0}\right\|_{b / 2}^{2} .
$$

Now we consider the case $1<s<b / 2$.

Taking $g=h^{n+1}$ in (3.1) and using the identity (3.5), we get

$$
\begin{aligned}
& \frac{1}{2 \Delta t}\left(\left\|h^{n+1}\right\|_{s}^{2}-\left\|h^{n}\right\|_{s}^{2}+\left\|h^{n+1}-h^{n}\right\|_{s}^{2}\right) \\
& \quad+\frac{1}{\mathrm{De}}\left\|\nabla h^{n+1}\right\|_{s}^{2}+\frac{b-2 s}{\mathrm{De}}\left(\frac{\boldsymbol{q}}{1-|\boldsymbol{q}|^{2}} h^{n+1}, \nabla h^{n+1}\right)_{s} \\
& \leqslant\left(\boldsymbol{K} \cdot \boldsymbol{q} h^{n}, \nabla h^{n+1}\right)_{s} .
\end{aligned}
$$

Then, by using the Cauchy-Schwarz inequality on the right-hand side, we find

$$
\begin{gathered}
\frac{1}{2 \Delta t}\left(\left\|h^{n+1}\right\|_{s}^{2}-\left\|h^{n}\right\|_{s}^{2}+\left\|h^{n+1}-h^{n}\right\|_{s}^{2}\right)+\frac{1}{2 \operatorname{De}}\left\|\nabla h^{n+1}\right\|_{s}^{2} \\
+\frac{b-2 s}{\operatorname{De}}\left(\frac{\boldsymbol{q}}{1-|\boldsymbol{q}|^{2}} h^{n+1}, \nabla h^{n+1}\right)_{s} \leqslant \frac{c_{0}}{2}\left\|h^{n}\right\|_{s}^{2} .
\end{gathered}
$$

Thanks to the property (2.25), we have

$$
\gamma\left\|h^{n+1}\right\|_{s}^{2}+\frac{b-2 s}{\operatorname{De}}\left(\frac{\boldsymbol{q}}{1-|\boldsymbol{q}|^{2}} h^{n+1}, \nabla h^{n+1}\right)_{s} \geqslant \beta(\gamma)\left\|h^{n+1}\right\|_{s-2}^{2}>0
$$

for $\gamma>\gamma_{\min }, \beta(\gamma)=\frac{b-2 s}{\operatorname{De}} \lambda\left(\frac{\operatorname{De} \gamma}{b-2 s}\right)$. Combining the above two relations, we find

$$
\begin{aligned}
\frac{1}{2 \Delta t}\left(\left\|h^{n+1}\right\|_{s}^{2}-\left\|h^{n}\right\|_{s}^{2}+\left\|h^{n+1}-h^{n}\right\|_{s}^{2}\right) & +\frac{1}{2 \mathrm{De}}\left\|\nabla h^{n+1}\right\|_{s}^{2} \\
& +\beta(\gamma)\left\|h^{n+1}\right\|_{s-2}^{2}-\gamma\left\|h^{n+1}\right\|_{s}^{2} \leqslant \frac{c_{0}}{2}\left\|h^{n}\right\|_{s}^{2},
\end{aligned}
$$

which we can rewrite as

$$
\begin{aligned}
(1-2 \gamma \Delta t)\left\|h^{n+1}\right\|_{s}^{2}+\left\|h^{n+1}-h^{n}\right\|_{s}^{2} & +\frac{\Delta t}{\mathrm{De}}\left\|\nabla h^{n+1}\right\|_{s}^{2} \\
& +2 \beta(\gamma) \Delta t\left\|h^{n+1}\right\|_{s-2}^{2} \leqslant\left(1+c_{0} \Delta t\right)\left\|h^{n}\right\|_{s}^{2} .
\end{aligned}
$$


Assuming $\Delta t<1 / 6 \gamma$, and dividing by $(1-2 \gamma \Delta t)$ on both sides of the above inequality, we obtain

$$
\begin{aligned}
& \left\|h^{n+1}\right\|_{s}^{2}+\left\|h^{n+1}-h^{n}\right\|_{s}^{2}+\frac{\Delta t}{\mathrm{De}}\left\|\nabla h^{n+1}\right\|_{s}^{2}+2 \beta(\gamma) \Delta t\left\|h^{n+1}\right\|_{s-2}^{2} \\
& \quad \leqslant \frac{1+c_{0} \Delta t}{1-2 \gamma \Delta t}\left\|h^{n}\right\|_{s}^{2} \\
& \left.\quad \leqslant\left(1+\left(3 c_{0} / 2+3 \gamma\right) \Delta t\right)\right)\left\|h^{n}\right\|_{s}^{2} .
\end{aligned}
$$

Then, summing up the above for $n=0,1, \ldots, m$, and denoting $c_{1}=3 c_{0} / 2+3 \gamma$, we get

$$
\begin{aligned}
\left\|h^{m+1}\right\|_{s}^{2}+\sum_{n=0}^{m}\left\|h^{n+1}-h^{n}\right\|_{s}^{2}+\frac{\Delta t}{\operatorname{De}} \sum_{n=0}^{m}\left\|\nabla h^{n+1}\right\|_{s}^{2} & +2 \beta(\gamma) \Delta t \sum_{n=0}^{m}\left\|h^{n+1}\right\|_{s-2}^{2} \\
& \leqslant\left\|h^{0}\right\|_{s}^{2}+c_{1} \Delta t \sum_{n=0}^{m}\left\|h^{n}\right\|_{s}^{2} .
\end{aligned}
$$

By applying a discrete Gronwall lemma, we can deduce that

$$
\begin{aligned}
\left\|h^{m+1}\right\|_{s}^{2}+\Delta t \sum_{n=0}^{m}\left\|\frac{h^{n+1}-h^{n}}{\sqrt{\Delta t}}\right\|_{s}^{2}+\frac{\Delta t}{\operatorname{De}} & \sum_{n=0}^{m}\left\|\nabla h^{n+1}\right\|_{s}^{2} \\
& +2 \beta(\gamma) \Delta t \sum_{n=0}^{m}\left\|h^{n+1}\right\|_{s-2}^{2} \leqslant e^{c_{1} m \Delta t}\left\|h^{0}\right\|_{s}^{2} .
\end{aligned}
$$

This completes the proof. $\quad \square$

We note that in the case of $s=b / 2$, Knezevic and Süli [14] considered a similar semi-implicit scheme and proved a similar stability result.

3.2. The stability of a second-order Crank-Nicolson scheme. Following a similar principle, we can easily construct second-order semi-implicit schemes which are unconditionally stable. We consider, as an example, the following Crank-Nicolson leapfrog scheme.

Given $h^{0}, h^{1} \in L^{2}\left(\Omega, M_{s}\right)$, for $n=1,2, \ldots$, find $h^{n+1}(\boldsymbol{q}) \in X_{s}(\Omega)$ such that

$$
\begin{aligned}
\left(\frac{h^{n+1}-h^{n-1}}{2 \Delta t}, g\right)_{s} & +\frac{b-2 s}{\operatorname{De}}\left(\frac{\boldsymbol{q}}{1-|\boldsymbol{q}|^{2}} \frac{h^{n+1}+h^{n-1}}{2}, \nabla g\right)_{s} \\
& +\frac{1}{\operatorname{De}}\left(\nabla \frac{h^{n+1}+h^{n-1}}{2}, \nabla g\right)_{s}=\left(K \cdot \boldsymbol{q} \cdot h^{n}, \nabla g\right)_{s} .
\end{aligned}
$$

Note that the above scheme needs two initial conditions $h^{0}$ and $h^{1}$ at $t=0$ and $t=\Delta t$, respectively. Given $h^{0}$, we can compute $h^{1}$ from the first-order scheme (3.1) without degrading the overall second-order accuracy.

Theorem 3.2. Let $1<s \leq b / 2, h^{0}, h^{1} \in L^{2}\left(\Omega, M_{s}\right)$, and $\gamma_{\min }$ be as defined in (2.26). Then, for $\Delta t>0$ when $s=b / 2$, and for $0<\Delta t<1 / \gamma_{\min }$ when $1<s<b / 2$, the scheme (3.6) admits a unique solution $h^{n+1}(\boldsymbol{q}) \in X_{s}(\Omega)$ for $n=1,2, \cdot$. Moreover, we have

$$
\left\|h^{m}\right\|_{s}^{2}+\left\|h^{m+1}\right\|_{s}^{2}+\frac{2 \Delta t}{\operatorname{De}} \sum_{n=1}^{m}\left\|\nabla \frac{h^{n+1}+h^{n-1}}{2}\right\|_{s-2}^{2} \leqslant e^{c_{0} m \Delta t}\left(\left\|h^{0}\right\|_{s}^{2}+\left\|h^{1}\right\|_{s}^{2}\right)
$$


for $s=b / 2$ with any $\Delta t$ and

$$
\begin{aligned}
&\left\|h^{m}\right\|_{s}^{2}+\left\|h^{m+1}\right\|_{s}^{2}+4 \beta(\gamma) \Delta t \sum_{n=1}^{m}\left\|\frac{h^{n+1}+h^{n-1}}{2}\right\|_{s-2}^{2}+\frac{2 \Delta t}{\mathrm{De}} \sum_{n=1}^{m}\left\|\nabla \frac{h^{n+1}+h^{n-1}}{2}\right\|_{s}^{2} \\
& \leqslant e^{c_{2} m \Delta t}\left(\left\|h^{0}\right\|_{s}^{2}+\left\|h^{1}\right\|_{s}^{2}\right)
\end{aligned}
$$

for $1<s<b / 2$ with $\Delta t<1 / 6 \gamma$ and $\gamma>\gamma_{\min }$. Here $c_{0}=\operatorname{De}|\mathbf{K}|_{\infty}^{2}, c_{2}=$ $\max \left\{6 \gamma, 3 c_{0}\right\}, \beta(\gamma)=\frac{b-2 s}{\mathrm{De}} \lambda\left(\frac{\mathrm{De} \gamma}{b-2 s}\right)$.

Proof. We shall only prove the case $1<s<b / 2$. The proof for $s=b / 2$ is similar.

Taking $g=\frac{h^{n+1}+h^{n-1}}{2}$ in (3.6), we get

$$
\begin{aligned}
& \frac{1}{4 \Delta t}\left(\left\|h^{n+1}\right\|_{s}^{2}-\left\|h^{n-1}\right\|_{s}^{2}\right)+\frac{1}{\mathrm{De}}\left\|\nabla \frac{h^{n+1}+h^{n-1}}{2}\right\|_{s}^{2} \\
& \quad+\frac{b-2 s}{\operatorname{De}}\left(\frac{\boldsymbol{q}}{1-|\boldsymbol{q}|^{2}} \frac{h^{n+1}+h^{n-1}}{2}, \nabla \frac{h^{n+1}+h^{n-1}}{2}\right)_{s}=\left(K \cdot \boldsymbol{q} \cdot h^{n}, \nabla \frac{h^{n+1}+h^{n-1}}{2}\right)_{s} .
\end{aligned}
$$

Using the Cauchy-Schwarz inequality, we find

$$
\left(K \cdot \boldsymbol{q} \cdot h^{n}, \nabla \frac{h^{n+1}+h^{n-1}}{2}\right)_{s} \leqslant \frac{\mathrm{De}}{2}|K|_{\infty}^{2}\left\|h^{n}\right\|_{s}^{2}+\frac{1}{2 \mathrm{De}}\left\|\nabla \frac{h^{n+1}+h^{n-1}}{2}\right\|_{s}^{2} .
$$

For the FENE potential term, we derive from the inequality (2.25) that

$$
\begin{aligned}
\beta(\gamma)\left\|\frac{h^{n+1}+h^{n-1}}{2}\right\|_{s-2}^{2} \leqslant & \frac{b-2 s}{\operatorname{De}}\left(\frac{\boldsymbol{q}}{1-|\boldsymbol{q}|^{2}} \frac{h^{n+1}+h^{n-1}}{2}, \nabla \frac{h^{n+1}+h^{n-1}}{2}\right)_{s} \\
& +\gamma\left\|\frac{h^{n+1}+h^{n-1}}{2}\right\|_{s}^{2}
\end{aligned}
$$

for $\gamma>\gamma_{\min }, \beta(\gamma)=\frac{b-2 s}{\operatorname{De}} \lambda\left(\frac{\operatorname{De} \gamma}{b-2 s}\right)>0$. Combining the three estimates above, we get

$$
\begin{aligned}
\frac{1}{4 \Delta t}\left(\left\|h^{n+1}\right\|_{s}^{2}-\left\|h^{n-1}\right\|_{s}^{2}\right) & +\beta(\gamma)\left\|\frac{h^{n+1}+h^{n-1}}{2}\right\|_{s-2}^{2}+\frac{1}{2 \operatorname{De}}\left\|\nabla \frac{h^{n+1}+h^{n-1}}{2}\right\|_{s}^{2} \\
& \leqslant \gamma\left\|\frac{h^{n+1}+h^{n-1}}{2}\right\|_{s}^{2}+\frac{\mathrm{De}}{2}|\boldsymbol{K}|_{\infty}^{2}\left\|h^{n}\right\|_{s}^{2},
\end{aligned}
$$

which we can rewrite as

$$
\begin{gathered}
(1-2 \gamma \Delta t)\left\|h^{n+1}\right\|_{s}^{2}+4 \beta(\gamma) \Delta t\left\|\frac{h^{n+1}+h^{n-1}}{2}\right\|_{s-2}^{2}+\frac{2 \Delta t}{\operatorname{De}}\left\|\nabla \frac{h^{n+1}+h^{n-1}}{2}\right\|_{s}^{2} \\
\leqslant(1+2 \gamma \Delta t)\left\|h^{n-1}\right\|_{s}^{2}+2 \operatorname{De}|\boldsymbol{K}|_{\infty}^{2} \Delta t\left\|h^{n}\right\|_{s}^{2} .
\end{gathered}
$$

Assuming $6 \gamma \Delta t<1$, we have

$$
\begin{aligned}
\left\|h^{n+1}\right\|_{s}^{2} & +4 \beta(\gamma) \Delta t\left\|\frac{h^{n+1}+h^{n-1}}{2}\right\|_{s-2}^{2}+\frac{2 \Delta t}{\operatorname{De}}\left\|\nabla \frac{h^{n+1}+h^{n-1}}{2}\right\|_{s}^{2} \\
& \leqslant \frac{1+2 \gamma \Delta t}{1-2 \gamma \Delta t}\left\|h^{n-1}\right\|_{s}^{2}+\frac{2 \operatorname{De}|K|_{\infty}^{2} \Delta t}{1-2 \gamma \Delta t}\left\|h^{n}\right\|_{s}^{2} \\
& \leqslant(1+6 \gamma \Delta t)\left\|h^{n-1}\right\|_{s}^{2}+3 \operatorname{De}|K|_{\infty}^{2} \Delta t\left\|h^{n}\right\|_{s}^{2} .
\end{aligned}
$$


Then, summing up the above for $n=1, \ldots, m$, we get

$$
\begin{gathered}
\left\|h^{m}\right\|_{s}^{2}+\left\|h^{m+1}\right\|_{s}^{2}+4 \beta(\gamma) \Delta t \sum_{n=1}^{m}\left\|\frac{h^{n+1}+h^{n-1}}{2}\right\|_{s-2}^{2}+\frac{2 \Delta t}{\operatorname{De}} \sum_{n=1}^{m}\left\|\nabla \frac{h^{n+1}+h^{n-1}}{2}\right\|_{s}^{2} \\
\leqslant\left\|h^{0}\right\|_{s}^{2}+\left\|h^{1}\right\|_{s}^{2}+6 \gamma \Delta t \sum_{n=1}^{m}\left\|h^{n-1}\right\|_{s}^{2}+3 \operatorname{De}|K|_{\infty}^{2} \Delta t \sum_{n=1}^{m}\left\|h^{n}\right\|_{s}^{2} \\
\leqslant\left\|h^{0}\right\|_{s}^{2}+\left\|h^{1}\right\|_{s}^{2}+c_{2} \Delta t \sum_{n=1}^{m}\left(\left\|h^{n-1}\right\|_{s}^{2}+\left\|h^{n}\right\|_{s}^{2}\right),
\end{gathered}
$$

where $c_{2}=\max \left\{6 \gamma, 3 \mathrm{De}|K|_{\infty}^{2}\right\}$. We can then deduce by using a discrete Gronwall lemma that

$$
\begin{gathered}
\left\|h^{m}\right\|_{s}^{2}+\left\|h^{m+1}\right\|_{s}^{2}+4 \beta(\gamma) \Delta t \sum_{n=1}^{m}\left\|\frac{h^{n+1}+h^{n-1}}{2}\right\|_{s-2}^{2}+\frac{2 \Delta t}{\operatorname{De}} \sum_{n=1}^{m}\left\|\nabla \frac{h^{n+1}+h^{n-1}}{2}\right\|_{s}^{2} \\
\leqslant e^{c_{2} m \Delta t}\left(\left\|h^{0}\right\|_{s}^{2}+\left\|h^{1}\right\|_{s}^{2}\right) .
\end{gathered}
$$

4. Fast spectral algorithms in two dimensions. In the two-dimensional case, $\boldsymbol{q}=\left(q_{1}, q_{2}\right)^{t} \in \Omega=\left\{\left(q_{1}, q_{2}\right): q_{1}^{2}+q_{2}^{2}<1\right\}, f(t, \boldsymbol{q})=f\left(t, q_{1}, q_{2}\right)$, and

$$
\boldsymbol{K}=\left(\nabla_{x} \boldsymbol{u}\right)^{t}=\left(\begin{array}{ll}
k_{11} & k_{12} \\
k_{21} & k_{22}
\end{array}\right) \quad \text { with } \quad k_{11}+k_{22}=0 .
$$

Denoting $\alpha=\kappa_{11}, \gamma=\left(\kappa_{12}-\kappa_{21}\right) / 2, \beta=\left(\kappa_{12}+k_{21}\right) / 2$, and $\varepsilon=\sqrt{\alpha^{2}+\beta^{2}}, \cos \varphi=$ $\alpha / \varepsilon, \sin \varphi=\beta / \varepsilon$, we have

$$
\begin{aligned}
& \boldsymbol{D}=\frac{\boldsymbol{K}+\boldsymbol{K}^{t}}{2}=\left(\begin{array}{ll}
\alpha & \beta \\
\beta & -\alpha
\end{array}\right)=\left(\begin{array}{ll}
\varepsilon & 0 \\
0 & -\varepsilon
\end{array}\right)\left(\begin{array}{ll}
\cos \varphi & \sin \varphi \\
-\sin \varphi & \cos \varphi
\end{array}\right), \\
& \boldsymbol{W}=\frac{\boldsymbol{K}-\boldsymbol{K}^{t}}{2}=\left(\begin{array}{ll}
0 & \gamma \\
-\gamma & 0
\end{array}\right) .
\end{aligned}
$$

Since the domain is a disk, it is convenient to use polar coordinates. Let $q_{1}=$ $r \cos \theta, q_{2}=r \cos \theta, h\left(t, q_{1}, q_{2}\right)=h(t, r \cos \theta, r \sin \theta)$. The weak formulation (2.12) is converted to the following. Find $h \in L^{2}\left(0, T ; X_{s}(\Sigma)\right) \cap L^{\infty}\left(0, T ; L^{2}\left(\Sigma, M_{s}\right)\right)$ such that $h(0, \cdot)=h_{0}(\cdot) \in L^{2}\left(\Sigma, M_{s}\right)$ and

$$
\begin{aligned}
\left(\frac{\partial h}{\partial t}, g\right)_{s} & +\frac{1}{\mathrm{De}}\left(\partial_{r} h, \partial_{r} g\right)_{s}+\frac{1}{\mathrm{De}}\left(\frac{1}{r^{2}} \partial_{\theta} h, \partial_{\theta} g\right)_{s}+\frac{b-2 s}{\mathrm{De}}\left(h, \frac{r}{1-r^{2}} \partial_{r} g\right)_{s} \\
& =\gamma\left(h, \partial_{\theta} g\right)_{s}+\varepsilon\left(h,\left[\cos (2 \theta-\varphi) r \partial_{r} g-\sin (2 \theta-\varphi) \partial_{\theta} g\right]\right)_{s} \quad \forall g \in X_{s}(\Sigma)
\end{aligned}
$$

where $(r, \theta) \in \Sigma:=[0,1] \times[0,2 \pi),(h, g)_{s}=\int_{\Sigma} h g\left(1-r^{2}\right)^{s} r \mathrm{~d} r \mathrm{~d} d \theta, X_{s}(\Sigma)=\{f:$ $\left.f(r, \theta)=g(r \cos \theta, r \sin \theta), g \in X_{s}(\Omega)\right\}$.

Due to the polar coordinate transform, the solution of (4.1) usually satisfies more pole conditions than what is minimally required in $X_{s}(\Sigma)$. More precisely, we can consider the following conditions at the pole:

(i) $h(\cdot, \theta)=h(\cdot, \theta+\pi)$ - the two ends of the dumbbells are indistinguishable;

(ii) $\left.\frac{\partial h}{\partial \theta}\right|_{r=0}=0$-required for the $C^{0}$ continuity (in the original variable $\boldsymbol{q}$ ) at the origin; 
(iii) $\left.\frac{\partial h}{\partial r}\right|_{r=0}=0$-required for the $C^{1}$ regularity (in the original variable $\boldsymbol{q}$ ) at the origin;

(iv) $h$ is $C^{\infty}$ (in the original variable $\boldsymbol{q}$ ) at the origin.

Since the variable change $f=h M_{s}$ does not affect the regularities at the pole, the above pole conditions for $h$ also apply to the CDF $f$. We note that only condition (ii) is essential with respect to the weak formulation (cf. [25]). It is relatively easy to construct efficient spectral schemes if we impose only the essential pole condition. However, one may get a more accurate solution if more pole conditions are imposed. Below, we propose two spectral algorithms imposing two sets of pole conditions, respectively: (a) conditions (i), (ii), and (iii); and (b) conditions (i), (ii), (iii), and (iv). The algorithm using pole condition set (a) is described in section 4.1, and the algorithm using pole condition set (b) is described in section 4.2.

Before discretizing (4.1) in space, we apply another mapping,

$$
r^{2}=\frac{p+1}{2},
$$

which converts the interval $[0,1]$ of $r$ to $[-1,1]$ of $p$. The mapping has been used in the past $[20,7]$. This mapping enjoys two main advantages: (i) the Gauss quadrature points are much less scattered around the pole and (ii) the polynomial degree in the weight function is reduced by half. With this mapping and setting $\psi(t, p, \theta)=$ $h(t, r, \theta),(4.1)$ becomes

$$
\begin{aligned}
& \left(\frac{\partial \psi}{\partial t}, g\right)_{s}+\frac{8}{\operatorname{De}}\left((1+p) \partial_{p} \psi, \partial_{p} g\right)_{s}+\frac{2}{\operatorname{De}}\left(\frac{1}{1+p} \partial_{\theta} \psi, \partial_{\theta} g\right)_{s}+\frac{4(b-2 s)}{\operatorname{De}}\left(\psi, \frac{1+p}{1-p} \partial_{p} g\right)_{s} \\
& =\gamma\left(\psi, \partial_{\theta} g\right)_{s}+\varepsilon\left(\psi, 2 \cos (2 \theta-\varphi)(1+p) \partial_{p} g-\sin (2 \theta-\varphi) \partial_{\theta} g\right)_{s} \quad \forall g \in Y\left(\Sigma^{\prime}\right),
\end{aligned}
$$

where $\Sigma^{\prime}=[-1,1] \times[0,2 \pi), Y\left(\Sigma^{\prime}\right)=\left\{f(p, \theta)=g\left(\sqrt{\frac{p+1}{2}}, \theta\right), g \in X_{s}(\Sigma)\right\}$, and $(f, g)_{s}=$ $\int_{\Sigma^{\prime}} f g \frac{(1-p)^{s}}{2^{s}} \mathrm{~d} p \mathrm{~d} \theta$.

4.1. A fast Jacobi-Galerkin method with $C^{1}$ regularity (in $q$ ) at the pole. Now we are ready to discretize (4.3) in space. It is easy to see that a suitable set of basis functions for the $\theta$ direction is the set of real Fourier series. Taking into account pole condition (i), we should expand the solution of (4.3) in the $\theta$ direction as

$$
\psi(p, \theta)=\sum_{\nu=0,1} \sum_{k=\nu}^{\infty} a_{\nu, k}(p) e_{\nu, k}(\theta)
$$

where

$$
e_{\nu, k}= \begin{cases}1 / \sqrt{2 \pi}, & \nu=0, k=0 \\ \cos (2 k \theta) / \sqrt{\pi}, & \nu=0, k>0 \\ \sin (2 k \theta) / \sqrt{\pi}, & \nu=1, k \geqslant 1\end{cases}
$$

Obviously, pole condition (i) is automatically satisfied. Pole condition (ii), which is the essential condition, leads to $\partial \psi /\left.\partial \theta\right|_{p=-1}=0$, i.e.,

$$
a_{\nu, k}(-1)=0 \quad \text { for } k \geqslant 1 .
$$

Pole condition (iii) is satisfied thanks to the mapping (4.2) since $\left.\frac{\partial \psi}{\partial r}\right|_{r=0}=\left.\frac{\partial \psi}{\partial p} 4 r\right|_{r=0}=0$. 
On the other hand, it is not as obvious to determine what should be used as basis functions in the $r$ direction. Due to the weight function $\left(\frac{1-p}{2}\right)^{s}$ in the weak formulation, a usual Chebyshev- or Legendre-Galerkin method (cf. [23, 24]) would lead to system (mass and stiffness) matrices with bandwidth which grows as $s$ increases. Therefore, it is better to build the weight function into the weight of basis functions so that the bandwidth of system matrices does not increase as $s$ increases. Taking into account the change of variables and the orthogonality relation of the Jacobi polynomials (cf. Appendixes A.1 and A.2), we are led to choose

$$
\phi_{k, i}(p)= \begin{cases}J_{i}^{s-2,0}(p), & k=0 \\ (1+p) J_{i}^{s-2,0}(p), & k \geqslant 1\end{cases}
$$

where $J_{i}^{s-2,0}$ is the $i$ th degree Jacobi polynomial with index $(s-2,0)$ (cf. [27] and Appendix A.1).

Denote by $P_{N}$ all the polynomials of degree less than or equal to $N, X_{N}^{0}=P_{N}$, $X_{N}^{k}=\left\{\phi(p) \in P_{N+1}, \phi(-1)=0\right\}$, and

$$
Y_{N}^{M}=\operatorname{span}\left\{e_{\nu, k}(\theta) a_{\nu, k}(p), \nu=0,1 ; k=\nu, \ldots, M ; a_{\nu, k}(p) \in X_{N}^{k}\right\} .
$$

Then, the first Jacobi-Galerkin method (JG1), applied to the first-order semi-implicit discretization in time (3.1), is as follows. Find $\psi_{N M}^{n+1} \in Y_{N}^{M}$ such that

$$
\begin{aligned}
& \left(\frac{\psi_{N M}^{n+1}-\psi_{N M}^{n}}{\Delta t}, g\right)_{s}+\frac{8}{\mathrm{De}}\left((1+p) \partial_{p} \psi_{N M}^{n+1}, \partial_{p} g\right)+\frac{2}{\mathrm{De}}\left(\frac{1}{1+p} \partial_{\theta} \psi_{N M}^{n+1}, \partial_{\theta} g\right)_{s} \\
& \quad+\frac{4(b-2 s)}{\mathrm{De}}\left(\psi_{N M}^{n+1}, \frac{1+p}{1-p} \partial_{p} g\right)_{s} \\
& =\gamma\left(\psi_{N M}^{n}, \partial_{\theta} g\right)_{s}+\varepsilon\left(\psi_{N M}^{n}, 2 \cos (2 \theta-\varphi)(1+p) \partial_{p} g-\sin (2 \theta-\varphi) \partial_{\theta} g\right)_{s} \quad \forall g \in Y_{N}^{M} .
\end{aligned}
$$

Writing $\psi_{N M}^{n}=\sum_{\nu=0,1} \sum_{k=\nu}^{M} \sum_{i=0}^{N} b_{\nu, k, i}^{n} e_{\nu, k}(\theta) \phi_{k, i}(p)$ and letting $g=e_{\mu, l}(\theta) \phi_{l, j}(p)$ in (4.7), we get a sequence of one-dimensional linear equations.

For each $\mu=0,1$ and $l=\mu, \ldots, M$,

(4.8) $\sum_{i=0}^{N}\left(O_{l, i}^{l, j}+\frac{8 \Delta t}{\operatorname{De}} P_{l, i}^{l, j}+\frac{8 \Delta t l^{2}}{\operatorname{De}} Q_{l, i}^{l, j}+\frac{4(b-2 s) \Delta t}{\operatorname{De}} R_{l, i}^{l, j}\right) b_{\mu, l, i}^{n+1}=\operatorname{RHS}(\mu, l, j)$,

where

$\operatorname{RHS}(\mu, l, j)=O_{l, i}^{l, j} b_{\mu, l, i}^{n}+\gamma \Delta t 2 l(\mu-\nu) O_{l, i}^{l, j} b_{\nu, l, i}^{n}+\varepsilon \Delta t\left(2 U_{\nu, k}^{\mu, l} S_{k, i}^{l, j} b_{\nu, k, i}^{n}-V_{\nu, k}^{\mu, l} O_{k i}^{l, j} b_{\nu, k, i}^{n}\right)$,

with the usual notation for repeating indexes, and for each $k, l$, and

$$
\left\{\begin{array}{l}
O_{k, i}^{l, j}=\left(\phi_{k, i}, \phi_{l, j}\right)_{s} \\
P_{k, i}^{l, j}=\left((1+p) \phi_{k, i}^{\prime}, \phi_{l, j}^{\prime}\right)_{s} \\
Q_{k, i}^{l, j}=\left(\frac{1}{1+p} \phi_{k, i}, \phi_{l, j}\right)_{s} \\
R_{k, i}^{l, j}=\left(\frac{1+p}{1-p} \phi_{k, i}, \phi_{l, j}^{\prime}\right)_{s} \\
S_{k, i}^{l, j}=\left((1+p) \phi_{k, i}, \phi_{l, j}^{\prime}\right)_{s}
\end{array} \quad \forall 0 \leq i, j \leq N,\right.
$$




$$
U_{\nu, k}^{\mu, l}=\left(\cos (2 \theta-\varphi) e_{\nu, k}, e_{\mu, l}\right), \quad V_{\nu, k}^{\mu, l}=\left(\sin (2 \theta-\varphi) e_{\nu, k}, e_{\mu, l}^{\prime}\right) .
$$

Thanks to (4.6), we only need to evaluate the matrices in (4.10) for $k, l=0,1$ since $O_{0}^{l}=O_{0}^{1} \forall l>1$, etc. For each $k, l=0,1$, the matrices $O_{k}^{l}, P_{k}^{l}, Q_{k}^{l}, R_{k}^{l}, S_{k}^{l}$ defined in (4.10) are all sparse with the number of nonzero diagonals equal to $9,7,7,7$, and 9, respectively (see the proof in Appendix A.2), thanks to the orthogonality of the Jacobi polynomials. Meanwhile, $U, V$ are tridiagonal matrices.

We now comment briefly on the computational cost for solving all the linear systems in (4.8). The storage for all the matrices is about $20 N M$. The computational cost for evaluating RHS is about $90 M N$, and the cost for solving all these linear systems with nine nonzero diagonals is about $18 M N$. So the total storage requirement is about $20 N M$, and the total computational cost at each time step is about $108 M N$. Thus, this algorithm has optimal computational complexity. It is obvious that this Fourier-Jacobi method applied to the second-order time discretization (cf. (3.6)) has the same computational complexity.

Note that the algorithm proposed in $[15,3]$ involves an LU decomposition for dense matrices, so the computational cost is $\mathcal{O}\left(8 M^{3} N^{3}\right)$ for initial setup and then $\mathcal{O}\left(4 M^{3} N^{2}\right)$ for every time step. The algorithm proposed in [20] has a slightly better computational complexity: $\mathcal{O}\left(8 M^{3} N^{3}\right)$ for initial setup and $\mathcal{O}\left(4 M^{2} N^{2}\right)$ for every time step. Hence, our algorithms are much faster than existing algorithms for moderate and large $M, N$. We emphasize that the key reasons for the efficiency of our algorithms are (i) we use semi-implicit schemes which treat the flow-transport term explicitly, an approach that allows us to reduce the two-dimensional problem to a sequence of onedimensional problems; and (ii) we construct basis functions using Jacobi polynomials with proper index which minimize the bandwidth of the system matrices.

4.2. A fast Jacobi-Galerkin method with $C^{\infty}$ regularity (in $q$ ) at the pole. Let us first derive the conditions for the $C^{\infty}$ regularity at the pole. Consider the Fourier expansion (4.4) and the mapping (4.2),

$$
\psi(p, \theta)=\sum_{\nu=0,1} \sum_{k=\nu}^{\infty} a_{\nu, k}(p) e_{\nu, k}(\theta)=\sum_{\nu=0,1} \sum_{k=\nu}^{\infty} \hat{a}_{\nu, k}(r) e_{\nu, k}(\theta)=h(r, \theta) .
$$

Then, for $h$ to be $C^{\infty}$ at the origin, we need (cf. [22])

$$
\left.\frac{\mathrm{d}^{i}}{\mathrm{~d} r^{i}} \hat{a}_{\nu, k}(r)\right|_{r=0}=0, \quad i=0,1, \ldots, 2 k-1,
$$

which is equivalent to

$$
\hat{a}_{\nu, k}(r)=r^{2 k} \tilde{\alpha}_{\nu, k}(r) .
$$

By the mapping (4.2), the condition (4.12) becomes

$$
\hat{a}_{\nu, k}(r)=a_{\nu, k}(p)=(1+p)^{k} / 2^{k} \tilde{\alpha}_{\nu, k}(p(r)) .
$$

Therefore, the proper functional space for $a_{\nu, k}(p)$ is

$$
\tilde{X}^{k}=\left\{\phi: \phi(p)=(1+p)^{k} \tilde{\phi} \quad \text { with } \quad \tilde{\phi} \in C^{\infty}([-1,1])\right\}
$$

and a proper approximation space for $\tilde{X}^{k}$ is

$$
\tilde{X}_{N}^{k}=\left\{\phi: \phi(p)=(1+p)^{k} g(p) \quad \text { with } \quad g(p) \in P_{N-k}\right\} .
$$


Setting

$$
\tilde{Y}_{N}^{M}=\operatorname{span}\left\{e_{\nu, k}(\theta) a_{\nu, k}(p), \nu=0,1 ; k=\nu, \ldots, M ; a_{\nu, k}(p) \in \tilde{X}_{N}^{k}\right\},
$$

the second Jacobi-Galerkin method (JGinf) (with a first-order semi-implicit discretization in time; cf. (3.1)) is as follows. Find $\psi_{N M}^{n+1} \in \tilde{Y}_{N}^{M}$ such that

$$
\begin{aligned}
& \left(\frac{\psi_{N M}^{n+1}-\psi_{N M}^{n}}{\Delta t}, g\right)_{s}+\frac{8}{\operatorname{De}}\left((1+p) \partial_{p} \psi_{N M}^{n+1}, \partial_{p} g\right)_{s} \\
& \quad+\frac{2}{\operatorname{De}}\left(\frac{1}{1+p} \partial_{\theta} \psi_{N M}^{n+1}, \partial_{\theta} g\right)_{s}+\frac{4(b-2 s)}{\operatorname{De}}\left(\psi_{N M}^{n+1}, \frac{1+p}{1-p} \partial_{p} g\right)_{s} \\
& =\gamma\left(\psi_{N M}^{n}, \partial_{\theta} g\right)_{s}+\varepsilon\left(\psi_{N M}^{n}, 2 \cos (2 \theta-\varphi)(1+p) \partial_{p} g-\sin (2 \theta-\varphi) \partial_{\theta} g\right)_{s} \quad \forall g \in \tilde{Y}_{N}^{M} .
\end{aligned}
$$

Given a set of basis functions $\left\{\varphi_{k, i}(p), i=0, \ldots, N-k\right\}$ for $\tilde{X}_{N}^{k}$ and write

$$
\psi_{N M}^{n}=\sum_{\nu=0,1} \sum_{k=\nu}^{M} \sum_{i=0}^{N-k} b_{\nu, k, i}^{n} e_{\nu, k}(\theta) \varphi_{k, i}(p)
$$

in (4.16), we obtain a sequence of one-dimensional linear systems similar to (4.8) for the first Jacobi-Galerkin method.

For each $\mu=0,1$ and $l=\mu, \ldots, M$,

$$
\sum_{i=0}^{N-l}\left(O_{l, i}^{l, j}+\frac{8 \Delta t}{\operatorname{De}} P_{l, i}^{l, j}+\frac{8 \Delta t l^{2}}{\operatorname{De}} Q_{l, i}^{l, j}+\frac{4(b-2 s) \Delta t}{\operatorname{De}} R_{l, i}^{l, j}\right) b_{\mu, l, i}^{n+1}=\operatorname{RHS}(\mu, l, j) .
$$

Here the matrices $O_{k}^{l}, P_{k}^{l}, Q_{k}^{l}, R_{k}^{l}, S_{k}^{l}$ are obtained by using new bases $\varphi_{k, i}(p)$. In order to minimize the bandwidth of these matrices, we choose the basis functions to be

$$
\varphi_{0, i}(p)=J_{i}^{s-2,0}(p), \quad \varphi_{k, i}(p)=(1+p)^{k} J_{i}^{s-2,2 k-1}(p) \text { for } k>0 .
$$

It can be verified (cf. Appendix A.3) that the matrices $O_{k}^{l}, P_{k}^{l}, Q_{k}^{l}, R_{k}^{l}, S_{k}^{l}$ are all sparse with only seven nonzero diagonals. Therefore, these linear systems can be solved by a computational cost similar to (in fact slightly less than) that of the first JacobiGalerkin method.

Note that the approximation property of this Jacobi-Galerkin method with $C^{\infty}$ regularity is mathematically equivalent to the linear moments closure model proposed in $\mathrm{Du}$, Liu, and $\mathrm{Yu}[8]$ with $\psi_{\mathrm{eq}}=\left(1-|\boldsymbol{q}|^{2}\right)^{b / 2}$. Actually, each term in an $n$ th-order moment can be expressed as

$$
\int_{\Omega} f q_{1}^{n_{1}} q_{2}^{n_{2}} \mathrm{~d} q_{1} \mathrm{~d} q_{2}=\int_{\Omega} h M_{s} r^{n} \cos ^{n_{1}} \theta \sin ^{n_{2}} \theta r \mathrm{~d} r \mathrm{~d} \theta, \quad n_{1}+n_{2}=n .
$$

When $n$ is odd, the moment equals to zero because $f(\boldsymbol{q})=f(-\boldsymbol{q})$; when $n$ is even, one can verify that $r^{n} \cos ^{n_{1}} \theta \sin ^{n_{2}} \theta=\left(\frac{p+1}{2}\right)^{n / 2} P_{n / 2}(\cos 2 \theta, \sin 2 \theta) \in Y_{n / 2}^{n / 2}$. However, taking moments as degrees of freedom is not a good choice for a big $n$ due to bad conditioning. 
5. Numerical results. In this section, we present some numerical results using the proposed algorithms and investigate how the solution error depends on the value of $s$.

In order to verify the accuracy of the algorithms, we take the function given in (2.3) as the initial condition and choose a symmetric $\mathbf{K}$ so that the exact solution at steady state is given by (2.2). The spectral coefficients for initial value of $h$ are computed by the Gauss-Jacobi quadrature. We recall that the computed solution $\psi_{N M}$ is an approximation to the solution $\psi$ of (4.3). The approximation $f_{N M}$ for the $\mathrm{CDF} f$ is then given by

$$
f_{N M}(t, \boldsymbol{q})=h_{N M}(t, \boldsymbol{q}) M_{s}(\boldsymbol{q})=\psi_{N M}(t, p, \theta) M_{s}(\boldsymbol{q}),
$$

where $(p, \theta)$ are related to $\boldsymbol{q}$ through the polar transform and (4.2).

To measure the convergence rate, we define

$$
\left\|f-f_{N M}\right\|_{L_{\gamma}^{2}}^{2}=\int_{\Sigma}\left(\frac{1-p}{2}\right)^{2 s+\gamma}\left(\psi-\psi_{N M}\right)^{2} \mathrm{~d} p \mathrm{~d} \theta
$$

and

$$
E\left(L_{\gamma}^{2}\right)=\frac{\left\|f-f_{\mathrm{eq}}^{D}\right\|_{L_{\gamma}^{2}}}{\left\|f_{\mathrm{eq}}^{D}\right\|_{L_{\gamma}^{2}}}
$$

In Tables 5.1 and 5.2, we list, respectively, the errors and CPU time with different sets of $(M, N)$ for Case $1, s=b / 2=6$, De $=24, \gamma=0, \beta=0$, and $\alpha=1$, and for Case $2, s=b / 2=10, \mathrm{De}=40, \gamma=0, \beta=0$, and $\alpha=2$. All the computations are performed on a Dell Inspiron 530 with Intel Core Quad CPU Q6600 at $2.40 \mathrm{GHz}$; only one core is used.

The approximate steady state solutions for Cases 1 and 2 are plotted in Figure 5.1.

We observe from Tables 5.1 and 5.2 that the JGinf method uses about half the degrees of freedom (DOF) and half the CPU times of the JG1 method while having similar accuracy. This savings in DOF and CPU, while modest in this two-

TABLE 5.1

The approximation error of the steady state solution in Case $1 . \quad s=b / 2=6$, De $=24$, $\gamma=0, \beta=0$ with other parameters $\alpha=1, \Delta t=0.05$. The CPU times measured in seconds for 1000 time steps are shown in the last two columns.

\begin{tabular}{|c|c|c|c|c|c|c|c|}
\hline$M$ & $N$ & DOF/JG1 & DOF/JGinf & $E\left(L_{-s}^{2}\right) /$ JG1 & $E\left(L_{-s}^{2}\right) /$ JGinf & CPU /JG1 & CPU/JGinf \\
\hline 10 & 10 & 231 & 121 & $0.12346 \mathrm{E}-02$ & $0.23598 \mathrm{E}-02$ & 0.116 & 0.095 \\
\hline 15 & 15 & 495 & 256 & $0.39179 \mathrm{E}-05$ & $0.54751 \mathrm{E}-05$ & 0.244 & 0.140 \\
\hline 20 & 20 & 861 & 441 & $0.41173 \mathrm{E}-08$ & $0.48941 \mathrm{E}-08$ & 0.335 & 0.215 \\
\hline 25 & 25 & 1326 & 676 & $0.17060 \mathrm{E}-11$ & $0.18602 \mathrm{E}-11$ & 0.487 & 0.311 \\
\hline 30 & 30 & 1891 & 961 & $0.11704 \mathrm{E}-12$ & $0.12328 \mathrm{E}-13$ & 0.692 & 0.417 \\
\hline
\end{tabular}

TABLE 5.2

The approximation error of the steady state solution in Case $2 . \quad s=b / 2=10$, De $=40$, $\gamma=0, \beta=0, \alpha=2, \Delta t=0.05$. The CPU times measured in seconds for 1000 time steps are shown in the last two columns.

\begin{tabular}{|c|c|c|c|c|c|}
\hline$M$ & $N$ & $E\left(L_{-s}^{2}\right) / J G 1$ & $E\left(L_{-s}^{2}\right) /$ JGinf & CPU/JG1 & CPU/JGinf \\
\hline 10 & 10 & blow-up & blow-up & 0.167 & 0.094 \\
\hline 20 & 20 & $0.18019 \mathrm{E}-02$ & $0.79619 \mathrm{E}-02$ & 0.336 & 0.249 \\
\hline 30 & 30 & $0.10653 \mathrm{E}-05$ & $0.36857 \mathrm{E}-05$ & 0.706 & 0.433 \\
\hline 40 & 40 & $0.14300 \mathrm{E}-09$ & $0.33844 \mathrm{E}-09$ & 1.118 & 0.786 \\
\hline
\end{tabular}



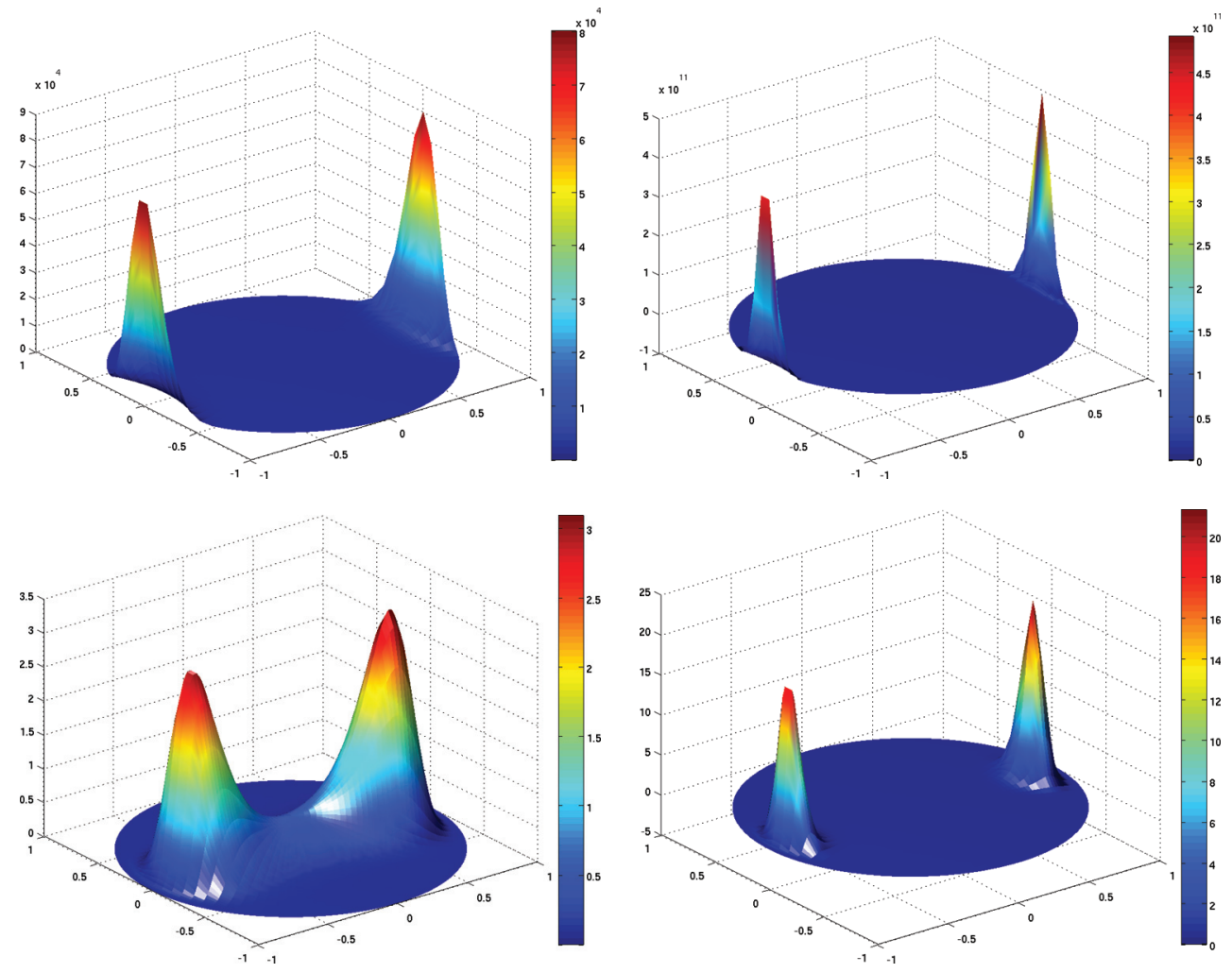

Fig. 5.1. Left: the steady solution of Case 1. Right: the steady solution of Case 2. Top: functions h. Bottom: functions $f$.

dimensional setting, could become significant in solving the coupled six-dimensional Navier-Stokes-Fokker-Planck equations. The JG1 method does have one advantage: it has a well-defined interpolation based on Fourier-Jacobi quadrature points, while such an interpolation operator is not available for the JGinf method.

To figure out which value of $s$ provides the best result, we carried out many simulations using different values of $b$, De, and $s$. Here we present three typical settings: Case $3, b=10, \mathrm{De}=20$; Case $4, b=72, \mathrm{De}=10, M=6, N=6$; and Case $5, b=72, \mathrm{De}=50, M=8, N=8$.

In all three cases, we let $\gamma=0, \beta=0, \alpha=1$, and $\Delta t=0.05$ and the JGinf method is used.

Case 3 is reported in Table 1 of [6]. We used the JGinf scheme with different values of $M, N$, and $s$ as used in [6]. We observe from Table 5.3 that our result is essentially identical to [6] and the numerical error for the polymer stress $\tau_{x x}$ is almost independent of the choice of $s$.

In Cases 4 and 5 , we choose different values of $s$. The numerical results of the solution error measured in the $L_{-s}^{2}$ norm and $L_{0}^{2}$ norm together with the error of the polymer stress $\tau_{x x}$ are presented in Table 5.4. We can clearly see that the polymer stress error is much smaller than the solution error. This is because the polymer stress depends only on the first few Jacobi-Fourier bases. For the smaller De number case, larger value of $s$ give better solution result. For the larger De number case, the best choice of $s$ is not $b / 2$. Some integer that is close to 28 will be the optimal choice of $s$. 
TABLE 5.3

The JGinf approximation error of the steady state polymer stress $\tau_{x x}$ in Case 3 with different values of $M, N$, and s. Note that the exact value reported in [6] is 9.37242277773, which is equal to the stress calculated using (1.4) multiplied by $\frac{b+4}{b}$.

\begin{tabular}{|r|c|c|c|c|}
\hline$M$ & $N$ & $E(s=2)$ in $[6]$ & $E(s=2)$ & $E(s=5)$ \\
\hline 5 & 11 & $3.7 \mathrm{E}-05$ & $3.69798 \mathrm{E}-05$ & $3.69798 \mathrm{E}-05$ \\
\hline 6 & 13 & $2.2 \mathrm{E}-06$ & $2.15125 \mathrm{E}-06$ & $2.15125 \mathrm{E}-06$ \\
\hline 7 & 15 & $1.1 \mathrm{E}-07$ & $1.06085 \mathrm{E}-07$ & $1.06085 \mathrm{E}-07$ \\
\hline 8 & 17 & $4.5 \mathrm{E}-09$ & $4.50044 \mathrm{E}-09$ & $4.50039 \mathrm{E}-09$ \\
\hline 9 & 19 & $1.6 \mathrm{E}-10$ & $1.66261 \mathrm{E}-10$ & $1.66618 \mathrm{E}-10$ \\
\hline 10 & 21 & $5.5 \mathrm{E}-12$ & $5.39702 \mathrm{E}-12$ & $5.54568 \mathrm{E}-12$ \\
\hline
\end{tabular}

TABLE 5.4

The solution error and polymer stress error of the steady state solution for Case 4 (the left four columns, $\mathrm{De}=10$ ) and Case 5 (the right four columns, $\mathrm{De}=50$ ) with different values of $s$.

\begin{tabular}{|c|c|c|c||c|c|c|c|}
\hline$s$ & $E\left(L_{-s}^{2}\right)$ & $E\left(L_{0}^{2}\right)$ & $E\left(\tau_{x x}\right)$ & $s$ & $E\left(L_{-s}^{2}\right)$ & $E\left(L_{0}^{2}\right)$ & $E\left(\tau_{x x}\right)$ \\
\hline 02 & $1.830 \mathrm{E}-01$ & $1.711 \mathrm{E}-01$ & $1.3247 \mathrm{E}-01$ & 12 & $7.658 \mathrm{E}-03$ & $6.295 \mathrm{E}-03$ & $1.0676 \mathrm{E}-09$ \\
\hline 08 & $4.007 \mathrm{E}-02$ & $3.204 \mathrm{E}-02$ & $8.9732 \mathrm{E}-04$ & 20 & $9.420 \mathrm{E}-04$ & $7.333 \mathrm{E}-04$ & $7.7693 \mathrm{E}-11$ \\
\hline 16 & $3.078 \mathrm{E}-03$ & $2.139 \mathrm{E}-03$ & $2.3410 \mathrm{E}-07$ & 24 & $8.516 \mathrm{E}-04$ & $2.276 \mathrm{E}-04$ & $1.0722 \mathrm{E}-11$ \\
\hline 24 & $8.865 \mathrm{E}-05$ & $5.675 \mathrm{E}-05$ & $9.1268 \mathrm{E}-14$ & 28 & $1.703 \mathrm{E}-03$ & $8.898 \mathrm{E}-05$ & $2.1693 \mathrm{E}-12$ \\
\hline 32 & $4.397 \mathrm{E}-07$ & $2.670 \mathrm{E}-07$ & $1.0324 \mathrm{E}-14$ & 32 & $3.576 \mathrm{E}-03$ & $3.353 \mathrm{E}-04$ & $5.6351 \mathrm{E}-12$ \\
\hline 36 & $1.631 \mathrm{E}-07$ & $1.705 \mathrm{E}-08$ & $1.3322 \mathrm{E}-14$ & 36 & $1.677 \mathrm{E}-02$ & $1.627 \mathrm{E}-02$ & $1.7415 \mathrm{E}-10$ \\
\hline
\end{tabular}

The error of the stress doesn't depend on $s$ in Case 3 because the value of $b$ is very small in that example, $b / 2-s$ is less than 4 for all valid $s$, but the numbers of the polynomials used in each Fourier mode are more than 10. The factor $M_{b / 2-s}$ in the equilibrium solution $h_{e q}$ can be well approximated by a function in $Y_{N}^{M}$ with $N>10$. In Case $4, M=6, N=6$, when $s$ is very small, $b / 2-s$ can be very large, the factor $M_{b / 2-s}$ itself cannot be well approximated by a function in $Y_{6}^{6}$. The optimal value of $s$ is not $b / 2$ when both $b$ and De are very large (see Case 5 ) because if the whole factor $M_{b / 2}$ of the equilibrium solution $f_{e q}^{\mathrm{D}}$ is put into the weight, the remaining part $\exp (\mathrm{De} / 2 \mathbf{D}: \mathbf{q q})$ is hard to approximate by functions in the corresponding Galerkin space $Y_{N}^{M}$. Based on those observations, we conclude that the best value of $s$ should be the one that minimizes the projection error of projecting the interested solutions into the Galerkin approximation space. Opposite to the observation of [6], the stability doesn't play a role in the choice of the optimal value of $s$.

6. Concluding remarks. We conducted in this paper a mathematical and numerical analysis of the Fokker-Planck equation for FENE dumbbell model.

We introduced a new weighted weak formulation and proved its well-posedness in weighted Sobolev spaces. In particular, the new weighted formulation is valid for $1<s \leq b / 2$. We also constructed simple, easy-to-implement semi-implicit timediscretization schemes which treat the flow interaction term explicitly. These semiimplicit schemes allow us to reduce the two-dimensonal problem into a sequence of one-dimensional problems. We then constructed two fast Fourier-Jacobi spectralGalerkin algorithms by using real Fourier series and special Jacobi polynomials as bases.

The advantages of our weighted formulation and spectral-Galerkin algorithms include the following: (i) it allows initial conditions in a much larger function space; (ii) depending on the nondimensional parameters, one can choose a special value of $s$ (rather than $s=b / 2$ and $s=2$ ) to give a better numerical result; and (iii) our 
spectral-Galerkin algorithms give spectral accuracy with linear computational cost. Thus, the algorithms presented in this paper provide a solid first step toward our ultimate goal of directly solving the coupled five- and six-dimensional Navier-StokesFokker-Planck equations.

In a forthcoming paper [26], we plan to develop fast spectral algorithms for the three-dimensional case and carry out a rigorous error analysis for the fully discretized schemes in both two and three dimensions.

\section{Appendix A.}

A.1. Basic properties of the Jacobi polynomials. Jacobi polynomials $\left\{J_{i}^{\alpha, \beta}(p), i=0,1, \ldots\right\}$ are orthogonal polynomials with respect to weight $\omega^{\alpha, \beta}(p)=$ $(1-p)^{\alpha}(1+p)^{\beta}$. All the properties we list below can be found in [1]. For simplicity, we denote the integration $\int_{-1}^{1} \cdot, \cdot \omega^{\alpha, \beta}(p) \mathrm{d} p$ by $(\cdot, \cdot)_{\alpha, \beta}$.

1. The recurrence relation:

$$
a_{n}^{\alpha, \beta} p J_{n}^{\alpha, \beta}=J_{n+1}^{\alpha, \beta}+b_{n}^{\alpha, \beta} J_{n}^{\alpha, \beta}+c_{n}^{\alpha, \beta} J_{n-1}^{\alpha, \beta} .
$$

2. The orthogonality of the $\left\{J_{n}^{\alpha, \beta}\right\}$ :

$$
\left(J_{i}^{\alpha, \beta}, J_{j}^{\alpha, \beta}\right)_{\alpha, \beta}=0 \text { if } i \neq j .
$$

3. The orthogonality of $\left\{\frac{\mathrm{d}}{\mathrm{d} p} J_{n}^{\alpha, \beta}\right\}$ :

$$
\left(\frac{\mathrm{d}}{\mathrm{d} p} J_{i}^{\alpha, \beta}, \frac{\mathrm{d}}{\mathrm{d} p} J_{j}^{\alpha, \beta}\right)_{\alpha+1, \beta+1}=\left(\left(1-p^{2}\right) \frac{\mathrm{d}}{\mathrm{d} p} J_{i}^{\alpha, \beta}, \frac{\mathrm{d}}{\mathrm{d} p} J_{j}^{\alpha, \beta}\right)_{\alpha, \beta}=0 \text { if } i \neq j .
$$

4. The differential relations (cf. p. 783 in [1]):

$$
(2 n+\alpha+\beta)\left(1-p^{2}\right) \frac{\mathrm{d}}{\mathrm{d} p} J_{n}^{\alpha, \beta}=n[\alpha-\beta-(2 n+\alpha+\beta) p] J_{n}^{\alpha, \beta}+2(n+\alpha)(n+\beta) J_{n-1}^{\alpha, \beta} .
$$

5. The recurrence relation (cf. p. 782 in [1]):

$$
\begin{aligned}
\left(n+\frac{\alpha+\beta}{2}+1\right)(1+p) J_{n}^{\alpha, \beta+1} & =(n+\beta+1) J_{n}^{\alpha, \beta}+(n+1) J_{n+1}^{\alpha, \beta}, \\
(2 n+\alpha+\beta) J_{n}^{\alpha, \beta-1} & =(n+\alpha+\beta) J_{n}^{\alpha, \beta}+(n+\alpha) J_{n-1}^{\alpha, \beta} .
\end{aligned}
$$

A.2. The sparsity of matrices $O, P, Q, R, S$ for JG1. In the first JacobiGalerkin method (JG1), the basis functions are given by (4.6). For notational simplicity, we omit below the superscript from $J_{i}^{s-2,0}$ and denote $(f, g)_{s}=\int_{-1}^{1} f g(1-p)^{s} \mathrm{~d} p$.

- For matrix $O$ : by using properties 1 and 2 in A.1, we have

$$
\begin{aligned}
& -O_{0, i}^{0, j}=\left(\phi_{0, i}, \phi_{0, j}\right)_{s}=\left((1-p) J_{i},(1-p) J_{j}\right)_{s-2}=0 \quad \text { for }|i-j|>2, \\
& -O_{0, i}^{1, j}=\left(\phi_{0, i}, \phi_{1, j}\right)_{s}=\left((1-p) J_{i},\left(1-p^{2}\right) J_{j}\right)_{s-2}=0 \quad \text { for }|i-j|>3, \text { and } \\
& -O_{1, i}^{1, j}=\left(\phi_{1, i}, \phi_{1, j}\right)_{s}=\left(\left(1-p^{2}\right) J_{i},\left(1-p^{2}\right) J_{j}\right)_{s-2}=0 \quad \text { for }|i-j|>4 .
\end{aligned}
$$

- For matrix $P$,

- by using the properties 1,2 , and 3 , we find

$$
\begin{aligned}
P_{0, i}^{0, j} & =\left((1+p) \phi_{0, i}^{\prime}, \phi_{0, j}^{\prime}\right)_{s}=\left(\left(1-p^{2}\right) J_{i}^{\prime},(1-p) J_{j}^{\prime}\right)_{s-2} \\
& =0 \quad \text { for }|i-j|>2 ;
\end{aligned}
$$

- by using the properties $1,2,3$, and 4 , we find

$$
\begin{aligned}
P_{1, i}^{1, j} & =\left((1+p) \phi_{1, i}^{\prime}, \phi_{1, j}^{\prime}\right)_{s}=\left((1-p)^{2}(1+p)\left((1+p) J_{i}\right)^{\prime},\left((1+p) J_{j}\right)^{\prime}\right)_{s-2} \\
& =0 \quad \text { for }|i-j|>3 .
\end{aligned}
$$


- For matrix $Q$ : by using properties 1 and 2 , we have

$$
Q_{1, i}^{1, j}=\left(\frac{1}{1+p} \phi_{1, i}, \phi_{1, j}\right)_{s}=\left((1+p) J_{i},(1-p)^{2} J_{j}\right)_{s-2}=0 \quad \text { for }|i-j|>3 .
$$

- For matrix $R$ : by using properties 1,2 , and 4 , we find

$-R_{0, i}^{0, j}=\left(\frac{1+p}{1-p} \phi_{0, i}, \phi_{0, j}^{\prime}\right)_{s}=\left(\left(1-p^{2}\right) J_{i}, J_{j}^{\prime}\right)_{s-2}=0 \quad$ for $|i-j|>1$ and

- $R_{1, i}^{1, j}=\left(\frac{1+p}{1-p} \phi_{1, i}, \phi_{1, j}^{\prime}\right)_{s}=\left(\left(1-p^{2}\right)(1+p) J_{i},\left((1+p) J_{j}\right)^{\prime}\right)_{s-2}=0 \quad$ for $\mid i-$ $j \mid>3$.

- For matrix $S$ : by using properties 1,2 , and 4 , we derive

$$
\begin{aligned}
& -S_{0, i}^{0, j}=\left((1+p) \phi_{0, i}, \phi_{0, j}^{\prime}\right)_{s}=\left(\left(1-p^{2}\right)(1-p) J_{i}, J_{j}^{\prime}\right)_{s-2}=0 \quad \text { for }|i-j|>2, \\
- & S_{0, i}^{1, j}=\left((1+p) \phi_{0, i}, \phi_{1, j}^{\prime}\right)_{s}=\left(\left(1-p^{2}\right)(1-p) J_{i},\left((1+p) J_{j}\right)^{\prime}\right)_{s-2}= \\
& 0 \quad \text { for }|i-j|>3, \\
- & S_{1, i}^{0, j}=\left((1+p) \phi_{1, i}, \phi_{0, j}^{\prime}\right)_{s}=\left(\left(1-p^{2}\right)\left(1-p^{2}\right) J_{i}, J_{j}^{\prime}\right)_{s-2}=0 \quad \text { for }|i-j|>3, \\
- & S_{1, i}^{1, j}=\left((1+p) \phi_{1, i}, \phi_{1, j}^{\prime}\right)_{s}=\left(\left(1-p^{2}\right)\left(1-p^{2}\right) J_{i},\left((1+p) J_{j}\right)^{\prime}\right)_{s-2}= \\
& 0 \quad \text { for }|i-j|>4 .
\end{aligned}
$$

A.3. The sparsity of matrices $O, P, Q, R, S$ for JGinf. In the second JacobiGalerkin method (JGinf), the basis functions are given by (4.18). For notational simplicity, we omit below the superscript from $J_{i}^{s-2,2 k-1}$. We star with the case $k=l$. For $k=l=0$, the matrices are exactly the same as in JG1. The cases $k=l \geq 1$ are given below.

- For matrix $O$,

$$
O_{k, i}^{k, j}=\left(\varphi_{k, i}, \varphi_{k, j}\right)_{s}=\left((1-p)^{2}(1+p) J_{i}, J_{j}\right)_{s-2,2 k-1}=0 \quad \text { for }|i-j|>3 .
$$

- For matrix $P$,

$$
\begin{aligned}
P_{k, i}^{k, j}= & \left((1+p) \varphi_{k, i}^{\prime}, \varphi_{k, j}^{\prime}\right)_{s} \\
= & \left((1-p)^{2}(1+p)\left((1+p)^{k} J_{i}\right)^{\prime},\left((1+p)^{k} J_{j}\right)^{\prime}\right)_{s-2} \\
= & \left((1-p)^{2}\left[k J_{i}+(1+p) J_{i}^{\prime}\right],\left[k J_{j}+(1+p) J_{j}^{\prime}\right]\right)_{s-2,2 k-1} \\
= & k^{2}\left((1-p)^{2} J_{i}, J_{j}\right)_{s-2,2 k-1}+k\left((1+p) J_{i},(1-p)^{2} J_{j}^{\prime}\right)_{s-2,2 k-1} \\
& +k\left((1-p)^{2} J_{i}^{\prime},(1+p) J_{j}\right)_{s-2,2 k-1}+\left(\left(1-p^{2}\right) J_{i}^{\prime},\left(1-p^{2}\right) J_{j}^{\prime}\right)_{s-2,2 k-1} \\
= & 0 \quad \text { for }|i-j|>2 .
\end{aligned}
$$

- For matrix $Q$,

$$
Q_{k, i}^{k, j}=\left(\frac{1}{1+p} \varphi_{k, i}, \varphi_{k, j}\right)_{s}=\left((1-p)^{2} J_{i}, J_{j}\right)_{s-2,2 k-1}=0 \quad \text { for }|i-j|>2 .
$$

- For matrix $R$,

$$
\begin{aligned}
R_{k, i}^{k, j} & =\left(\frac{1+p}{1-p} \varphi_{k, i}, \varphi_{k, j}^{\prime}\right)_{s} \\
& =\left((1-p)(1+p)^{k+1} J_{i},\left[(1+p)^{k} J_{j}\right]^{\prime}\right)_{s-2} \\
& =k\left(\left(1-p^{2}\right) J_{i}, J_{j}\right)_{s-2,2 k-1}+\left((1+p) J_{i},\left(1-p^{2}\right) J_{j}^{\prime}\right)_{s-2,2 k-1} \\
& =0 \quad \text { for }|i-j|>2 .
\end{aligned}
$$


Next, we consider the cases $k=l \pm 1$.

- For matrix $O$,

$$
O_{1, i}^{0, j}=\left(\varphi_{1, i}, \varphi_{0, j}\right)_{s}=\left((1+p) J_{i}^{s-2,1},(1-p)^{2} J_{j}^{s-2,0}\right)_{s-2}=0 \quad \text { for }|i-j|>3
$$

and

$$
\begin{aligned}
O_{l+1, i}^{l, j} & =\left(\varphi_{l+1, i}, \varphi_{l, j}\right)_{s} \\
& =\left((1-p)^{2}(1+p)^{l+1} J_{i}^{s-2,2 l+1},(1+p)^{l} J_{j}^{s-2,2 l-1}\right)_{s-2} \\
& =\left((1+p)^{2} J_{i}^{s-2,2 l+1},(1-p)^{2} J_{j}^{s-2,2 l-1}\right)_{s-2,2 l-1} \\
& =0 \quad \text { for }|i+1-j|>3, l \geq 1 .
\end{aligned}
$$

Due to the symmetry of $O$,

$$
Q_{l-1, i}^{l, j}=Q_{l, j}^{l-1, i}, \quad l \geq 1
$$

- For matrix $S$,

$$
\begin{aligned}
& S_{0, i}^{0, j}=\left((1+p) \varphi_{0, i}, \varphi_{0, j}^{\prime}\right)_{s}=\left(\left(1-p^{2}\right)(1-p) J_{i}, J_{j}^{\prime}\right)_{s-2}=0 \quad \text { for }|i-j|>2 \\
& S_{k, i}^{k, j}=\left((1+p) \varphi_{k, i}, \varphi_{k, j}^{\prime}\right)_{s} \\
& =\left((1-p)^{2}(1+p)^{k+1} J_{i},\left[(1+p)^{k} J_{j}\right]^{\prime}\right)_{s-2} \\
& =\left((1-p)^{2}(1+p) J_{i}, k J_{j}+(1+p) J_{j}^{\prime}\right)_{s-2,2 k-1} \\
& =0 \quad \text { for }|i-j|>3, k \geq 1 \\
& \quad S_{1, i}^{0, j}=\left((1+p) \varphi_{1, i}, \varphi_{0, j}^{\prime}\right)_{s}=\left((1+p)^{2} J_{i}^{s-2,1},(1-p)^{2} J_{j}^{\prime s-2,0}\right)_{s-2} \\
& \quad \text { for }|i-j|>3 \\
& S_{l+1, i}^{l, j} \quad\left((1+p) \varphi_{l+1, i}, \varphi_{l, j}^{\prime}\right)_{s} \\
& =\left((1-p)^{2}(1+p)^{l+2} J_{i}^{s-2,2 l+1},\left[(1+p)^{l} J_{j}^{s-2,2 l-1}\right]^{\prime}\right)_{s-2} \\
& =\left((1-p)^{2}(1+p)^{2} J_{i}^{s-2,2 l+1}, l J_{j}^{s-2,2 l-1}+(1+p) J_{j}^{\prime s-2,2 l-1}\right)_{s-2,2 l-1} \\
& =\left((1+p)^{2} J_{i}^{s-2,2 l+1}, l(1-p)^{2} J_{j}^{s-2,2 l-1}+(1-p)\left(1-p^{2}\right) J_{j}^{\prime s-2,2 l-1}\right)_{s-2,2 l-1} \\
& =0, \quad \text { for }|i+1-j|>3, l \geq 1 \\
& S_{0, i}^{1, j}=\left((1+p) \varphi_{0, i}, \varphi_{1, j}^{\prime}\right)_{s}=\left((1-p)^{2}(1+p) J_{i}^{s-2,0},\left[(1+p) J_{j}^{s-2,1}\right]_{s-2}^{\prime}\right. \\
& =0 \quad \text { for }|i-j|>3
\end{aligned}
$$




$$
\begin{aligned}
& S_{l-1, i}^{l, j} \\
& =\left((1+p) \varphi_{l-1, i}, \varphi_{l, j}^{\prime}\right)_{s} \\
& =\left((1-p)^{2}(1+p)^{l} J_{i}^{s-2,2 l-3},\left[(1+p)^{l} J_{j}^{s-2,2 l-1}\right]^{\prime}\right)_{s-2} \\
& =\left((1-p)^{2}(1+p)^{2} J_{i}^{s-2,2 l-3}, l J_{j}^{s-2,2 l-1}+(1+p) J_{j}^{s-2,2 l-1}\right)_{s-2,2 l-3} \\
& =\left(J_{i}^{s-2,2 l-3}, l(1-p)^{2}(1+p)^{2} J_{j}^{s-2,2 l-1}\right. \\
& \left.\quad+(1-p)(1+p)^{2}\left(1-p^{2}\right) J_{j}^{\prime s-2,2 l-1}\right)_{s-2,2 l-3} \\
& =0 \quad \text { for }|i-j-1|>3, l>1 .
\end{aligned}
$$

\section{REFERENCES}

[1] M. Abramowitz and I. A. Stegun, eds., Handbook of Mathematical Functions with Formulas, Graphs, and Mathematical Tables, National Bureau of Standards Appl. Math. Ser. 55, U.S. Department of Commerce, 1972.

[2] A. Arnold, J. A. Carrillo, and C. Manzini, Refined long-time asymptotics for some polymeric fluid flow models, Commun. Math. Sci., 8 (2010), pp. 763-782.

[3] J. Barrett, D. Knezevic, And E. Süli, Kinetic Models of Dilute Polymers: Analysis, Approximation and Computation, Nečas Center for Mathematical Modeling, Prague, 2009.

[4] J. W. BARRETT AND E. SüLI, Existence of global weak solutions to some regularized kinetic models for dilute polymers, Multiscale Model. Simul., 6 (2007), pp. 506-546.

[5] J. W. BARRETT AND E. SÜLI, Existence of global weak solutions to dumbbell models for dilute polymers with microscopic cut-off, Math. Models Methods Appl. Sci., 18 (2008), pp. 935971.

[6] C. Chauvière And A. Lozinski, Simulation of dilute polymer solutions using a Fokker-Planck equation, Comput. Fluids, 33 (2004), pp. 687-696.

[7] C. Chauvière And A. Lozinski, Simulation of complex viscoelastic flows using the FokkerPlanck equation: 3D FENE model, J. Non-Newtonian Fluid Mechanics, 122 (2004), pp. 201-214.

[8] Q. Du, C. LiU, AND P. Yu, FENE dumbbell model and its several linear and nonlinear closure approximations, Multiscale Model. Simul., 4 (2005), pp. 709-731.

[9] L. C. Evans, Partial Differential Equations, Grad. Stud. Math. 19, American Mathematical Society, Providence, RI, 1998.

[10] X.-J. FAN, Viscosity, first normal-stress coefficient, and molecular stretching in dilute polymer solutions, J. Non-Newtonian Fluid Mechanics, 17 (1985), pp. 125-144.

[11] M. Herrchen and H. C. Öttinger, A detailed comparison of various FENE dumbbell models, J. Non-Newtonian Fluid Mechanics, 68 (1997), pp. 17-42.

[12] B. Jourdain, C. Le Bris, T. Lelièvre, And F. Otto, Long-time asymptotics of a multiscale model for polymeric fluid flows, Arch. Ration. Mech. Anal., 181 (2006), pp. 97-148.

[13] B. Jourdain and T. LeLIÉvre, Mathematical analysis of a stochastic differential equation arising in the micro-macro modelling of polymeric fluids, in Probabilistic Methods in Fluids: Proceedings of the Swansea 2002 Workshop, Wales, UK, 2003, pp. 205-223.

[14] D. J. KNEZEVIC AND E. SÜLI, A heterogeneous alternating-direction method for a micro-macro dilute polymeric fluid model, ESAIM Math. Model. Numer. Anal., 43 (2009), pp. 11171156.

[15] D. J. KNEZEVIC AND E. SüLI, Spectral galerkin approximation of Fokker-Planck equations with unbounded drift, ESAIM Math. Model. Numer. Anal., 43 (2009), pp. 445-485.

[16] A. Kufner, Weighted Sobolev Spaces, John Wiley \& Sons, New York, 1985.

[17] T. Li And P. Zhang, Mathematical analysis of multi-scale models of complex fluids, Comm. Math. Sci., 5 (2007), pp. 1-51.

[18] J. L. Lions And E. Magenes, Non-Homogeneous Boundary Value Problems and Applications: Vol. 3, Springer-Verlag, New York, 1973.

[19] C. Liu And H. LiU, Boundary conditions for the microscopic FENE models, SIAM J. Appl. Math., 68 (2007), pp. 1304-1315. 
[20] A. Lozinski And C. Chauvière, A fast solver for Fokker-Planck equation applied to viscoelastic flows calculations: 2D FENE model, J. Comput. Phys., 189 (2003), pp. 607-625.

[21] N. MAsmoudi, Well-posedness for the FENE dumbbell model of polymeric flows, Commun. Pure Appl. Math., 61 (2008), pp. 1685-1714.

[22] T. Matsushima and P. S. Marcus, A spectral method for polar coordinates, J. Comput. Phys., 120 (1995), pp. 365-374.

[23] J. Shen, Efficient Spectral-Galerkin method I: Direct solvers of second- and fourth-order equations using Legendre polynomials, SIAM J. Sci. Comput., 15 (1994), p. 1489.

[24] J. ShEn, Efficient spectral-Galerkin method II: Direct solvers of second-and fourth-order equations using Chebyshev polynomials, SIAM J. Sci. Comput., 16 (1995), pp. 74-87.

[25] J. Shen, Efficient Spectral-Galerkin methods III: Polar and cylindrical geometries, SIAM J. Sci. Comput., 15 (1997), pp. 1489-1505.

[26] J. Shen And H. Yu, Spectral Methods for the FENE Fokker-Planck Equation II: Numerical Analysis and Optimal 3D Solver, preprint, 2012.

[27] G. Szegö, Orthogonal Polynomials, American Mathematical Society, Providence, RI, 1939.

[28] R. Temam, Navier-Stokes Equations and Nonlinear Functional Analysis, 2nd ed., CBMS-NSF Regional Conf. Ser. in Appl. Math., SIAM, Philadelphia, 1987.

[29] H. WANG, K. LI, AND P. Zhang, Crucial properties of the moment closure model FENE-QE, J. Non-Newtonian Fluid Mechanics, 150 (2008), pp. 80-92.

[30] H. R. WARNER, Kinetic theory and rheology of dilute suspensions of finitely extendible dumbbells, Indust. Engrg. Chemistry Fundamentals, 11 (1972), pp. 379-387.

[31] H. Zhang And P. Zhang, Local existence for the FENE-Dumbbell model of polymeric fluids, Arch. Ration. Mech. Anal., 181 (2006), pp. 373-400. 